\title{
NONEXISTENCE OF TRAVELING WAVES FOR A NONLOCAL GROSS-PITAEVSKII EQUATION
}

\author{
ANDRÉ DE LAIRE
}

\begin{abstract}
We consider a Gross-Pitaevskii equation with a nonlocal interaction potential. We provide sufficient conditions on the potential such that there exists a range of speeds in which nontrivial traveling waves do not exist.
\end{abstract}

\section{INTRODUCTION}

1.1. The problem. We consider finite energy traveling waves for the nonlocal Gross-Pitaevskii equation

$$
i \partial_{t} u-\Delta u-u\left(W *\left(1-|u|^{2}\right)\right)=0, \quad u(x, t) \in \mathbb{C}, x \in \mathbb{R}^{N}, t \in \mathbb{R} .
$$

Here $*$ denotes the convolution in $\mathbb{R}^{N}$ and $W$ is a real-valued even distribution. The aim of this work is to provide sufficient conditions on the potential $W$ such that these traveling waves are necessarily constant for a certain range of speeds. Equation (1.1) is Hamiltonian and its energy

$$
E(u(t))=\frac{1}{2} \int_{\mathbb{R}^{N}}|\nabla u(t)|^{2} d x+\frac{1}{4} \int_{\mathbb{R}^{N}}\left(W *\left(1-|u(t)|^{2}\right)\right)\left(1-|u(t)|^{2}\right) d x
$$

is formally conserved. A traveling wave of speed $c$ that propagates along the $x_{1}$-axis is a solution of the form

$$
u_{c}(x, t)=v\left(x_{1}-c t, x_{\perp}\right), \quad x_{\perp}=\left(x_{2}, \ldots, x_{N}\right) .
$$

Hence the profile $v$ satisfies

$$
i c \partial_{1} v+\Delta v+v\left(W *\left(1-|v|^{2}\right)\right)=0 \text { in } \mathbb{R}^{N}
$$

and by using complex conjugation, we can restrict us to the case $c \geq 0$. Note that any constant (complex-valued) function $v$ of modulus one verifies (NTWc), so that we refer to them as the trivial solutions.

Notice that, in the case that $W$ coincides with the Dirac delta function, (NTWc) reduces to the classical Gross-Pitaevskii equation

$$
i c \partial_{1} v+\Delta v+v\left(1-|v|^{2}\right)=0 \text { in } \mathbb{R}^{N} .
$$

Equation (TWC) has been intensively studied in the last years. We refer to [3] for a survey. From now on we suppose that $N \geq 2$ and we recall the following results.

2000 Mathematics Subject Classification. 35Q55; 35Q40; 35Q51; 35B65; 37K40; 37K05; $81 \mathrm{Q} 99$.

Key words and phrases. Nonlocal Schrödinger equation, Gross-Pitaevskii equation, Traveling waves, Pohozaev identities, Nonzero conditions at infinity.

The author is grateful to F. Béthuel for interesting and helpful discussions and to P. Gravejat for his collaboration in proving Proposition 5.3 
Theorem $1.1\left(\left[8,6,[16,[17))\right.\right.$. Let $v \in H_{\mathrm{loc}}^{1}\left(\mathbb{R}^{N}\right)$ be a finite energy solution of (TWC). Assume that one of the following cases hold

(i) $c=0$.

(ii) $c>\sqrt{2}$.

(iii) $N=2$ and $c=\sqrt{2}$.

Then $v$ is a constant function of modulus one.

Theorem 1.2 ([6, 5, 10, 4, 24]). There is some nonempty set $A \subset(0, \sqrt{2})$ such that for all $c \in A$ there exists a nonconstant finite energy solution of (TWc). Furthermore, assume that $N \geq 3$. Then there exists a nonconstant finite energy solution of (TWC) for all $0<c<\sqrt{2}$.

It would be reasonable to expect to generalize in some way these theorems to the nonlocal equation (NTWC). The aim of this paper is to investigate the analogue of Theorem 1.1 in the cases (i) and (ii), Before stating our precise results, we give some motivation about the critical speed.

1.2. Physical motivation. As explained in [13, (1.1) can be considered as a generalization of the equation

$$
i \hbar \partial_{t} \Psi(x, t)=\frac{\hbar^{2}}{2 m} \Delta \Psi(x, t)+\Psi(x, t) \int_{\mathbb{R}^{N}}|\Psi(y, t)|^{2} V(x-y) d y, \text { in } \mathbb{R}^{N} \times \mathbb{R},
$$

introduced by Gross [18] and Pitaevskii [27] to describe the kinetic of a weakly interacting Bose gas of bosons of mass $m$, where $\Psi$ is the wavefunction governing the condensate in the Hartree approximation and $V$ describes the interaction between bosons.

In the most typical approximation, $V$ is considered as a Dirac delta function. Then this model has applications in several areas of physics, such as superfluidity, nonlinear optics and Bose-Einstein condensation [21, 20, 23, 11]. It seems then natural to analyze equation (1.2) for more general interactions. Indeed, in the study of superfluidity, supersolids and Bose-Einstein condensation, different types of nonlocal potentials have been proposed [7, 2, 14, 29, 28, 22, 30, 12, 9, 1.

Let us now proceed formally and consider a constant function $u_{0}$ of modulus one. Since (1.1) is invariant by a change of phase, we can assume $u_{0}=1$. Then the linearized equation of (1.1) at $u_{0}$ is given by

$$
i \partial_{t} \tilde{u}-\Delta \tilde{u}+2 W * \operatorname{Re}(\tilde{u})=0 .
$$

Writing $\tilde{u}=\tilde{u}_{1}+i \tilde{u}_{2}$ and taking real and imaginary parts in (1.3), we get

$$
\begin{aligned}
-\partial_{t} \tilde{u}_{2}-\Delta \tilde{u}_{1}+2 W * \tilde{u}_{1} & =0, \\
\partial_{t} \tilde{u}_{1}-\Delta \tilde{u}_{2} & =0,
\end{aligned}
$$

from where we deduce that

$$
\partial_{t t}^{2} \tilde{u}-2 W *(\Delta \tilde{u})+\Delta^{2} \tilde{u}=0 .
$$

By imposing $\tilde{u}=e^{i(\xi \cdot x-w t)}, w \in \mathbb{R}, \xi \in \mathbb{R}^{N}$, as a solution of (1.4), we obtain the dispersion relation

$$
(w(\xi))^{2}=|\xi|^{4}+2 \widehat{W}(\xi)|\xi|^{2},
$$


where $\widehat{W}$ denotes the Fourier transform of $W$. Supposing that $\widehat{W}$ is positive and continuous at the origin, we get in the long wave regime, i.e. $\xi \sim 0$,

$$
w(\xi) \sim(2 \widehat{W}(0))^{1 / 2}|\xi| .
$$

Consequently, in this regime we can identify $(2 \widehat{W}(0))^{1 / 2}$ as the speed of sound waves (also called sonic speed), so that we set

$$
c_{s}(W)=(2 \widehat{W}(0))^{1 / 2} .
$$

The dispersion relation (1.5) was first observed by Bogoliubov [7] on the study of Bose-Einstein gas and under some physical considerations he established that the gas should move with a speed less than $c_{s}(W)$ to preserve its superfluid properties. From a mathematical point of view and comparing with Theorems 1.1 and 1.2, this encourages us to think that the nonexistence of a nontrivial solution of (NTWC) is related to the condition

$$
c>c_{s}(W) .
$$

Actually, in Subsection 1.4 we provide results in this direction and in Subsection 1.5 we specify the discussion for some explicit potentials $W$ which are physically relevant.

1.3. Hypotheses on $W$. Let us introduce the spaces $\mathcal{M}_{p, q}\left(\mathbb{R}^{N}\right)$ of tempered distributions $W$ such that the linear operator $f \mapsto W * f$ is bounded from $L^{p}\left(\mathbb{R}^{N}\right)$ to $L^{q}\left(\mathbb{R}^{N}\right)$. We will use the following hypotheses on $W$.

(H1) $W$ is a real-valued even temperated distribution.

(H2) $W \in \mathcal{M}_{2,2}\left(\mathbb{R}^{N}\right)$. Moreover, if $N \geq 4$,

$$
W \in \mathcal{M}_{N /(N-1), \infty}\left(\mathbb{R}^{N}\right) \cap \mathcal{M}_{2 N /(N-2), \infty}\left(\mathbb{R}^{N}\right) \cap \mathcal{M}_{2 N /(N-2), 2 N /(N-2)}\left(\mathbb{R}^{N}\right) .
$$

(H3) $\widehat{W}$ is differentiable a.e. on $\mathbb{R}^{N}$ and for all $j, k \in\{1, \ldots, N\}$ the map $\xi \rightarrow$ $\xi_{j} \partial_{k} \widehat{W}(\xi)$ is bounded and continuous a.e. on $\mathbb{R}^{N}$.

(H4) $\widehat{W} \geq 0$ a.e. on $\mathbb{R}^{N}$.

(H5) $\widehat{W}$ is of class $C^{2}$ in a neighborhood of the origin and $\widehat{W}(0)>0$.

Recall that the condition $W \in \mathcal{M}_{2,2}\left(\mathbb{R}^{N}\right)$ is equivalent to $\widehat{W} \in L^{\infty}\left(\mathbb{R}^{N}\right)$ (see e.g. [15]). Therefore (H4) makes sense provided that (H2) holds. It is proved in [13] that under the assumptions (H1), (H2) and (H4) the Cauchy problem for (1.1) with nonzero condition at infinity is globally well-posed. Actually, condition (1.7) is more restrictive that the one used in [13] in dimension $N \geq 4$, but we need it to ensure the regularity of solutions. More precisely, in Section 2 we prove that under the hypothesis (H2), the solutions of (NTWc) are smooth and satisfy

$$
|v(x)| \rightarrow 1, \nabla v(x) \rightarrow 0, \quad \text { as }|x| \rightarrow \infty .
$$

On the other hand, by Lemma 2.3. (1.7) is at least fulfilled for $W \in L^{1}\left(\mathbb{R}^{N}\right) \cap$ $L^{N}\left(\mathbb{R}^{N}\right)$.

Assumption (H2) also implies that $E(v)$ is finite in the energy space

$$
\mathcal{E}\left(\mathbb{R}^{N}\right)=\left\{\varphi \in H_{\text {loc }}^{1}\left(\mathbb{R}^{N}\right): 1-|\varphi|^{2} \in L^{2}\left(\mathbb{R}^{N}\right), \nabla \varphi \in L^{2}\left(\mathbb{R}^{N}\right)\right\} .
$$


Furthermore, if (H4) also holds, then by the Plancherel identity

$$
E(v)=\frac{1}{2} \int_{\mathbb{R}^{N}}|\nabla v|^{2}+\frac{1}{4(2 \pi)^{N}} \int_{\mathbb{R}^{N}} \widehat{W}\left|\widehat{1-|v|^{2}}\right|^{2} \geq 0 .
$$

In Subsection 1.5 we show several examples of distributions $W$ satisfying the conditions (H1) (H5)

\subsection{Statement of the results.}

Theorem 1.3. Assume that $W$ satisfies $(\mathrm{H} 1)(\mathrm{H} 5)$, Let $c>c_{s}(W)$ and suppose that there exist constants $\sigma_{1}, \ldots, \sigma_{N} \in \mathbb{R}$ such that

$$
\widehat{W}(\xi)+\alpha_{c} \sum_{k=2}^{N} \sigma_{k} \xi_{k} \partial_{k} \widehat{W}(\xi)-\sigma_{1} \xi_{1} \partial_{1} \widehat{W}(\xi) \geq 0 \text {, for a.a. } \xi \in \mathbb{R}^{N},
$$

and

$$
\sum_{k=2}^{N} \sigma_{k}+\min \left\{-\sigma_{1}-1, \frac{\sigma_{1}-1}{\alpha_{c}+2}, 2 \alpha_{c} \sigma_{j}+\sigma_{1}-1\right\} \geq 0,
$$

for all $j \in\{2, \ldots, N\}$, where $\alpha_{c}:=c^{2} /\left(c_{s}(W)\right)^{2}-1$. Then nontrivial solutions of (NTWC) in $\mathcal{E}\left(\mathbb{R}^{N}\right)$ do not exist.

To apply Theorem 1.3 we need to verify the existence of the constants $\sigma_{1}, \ldots, \sigma_{N}$ satisfying (1.8) and (1.9). To avoid this task, we provide two corollaries where the conditions for the nonexistence of traveling waves are expressed only in terms of $W$.

Corollary 1.4. Assume that $W$ satisfies $(\mathrm{H} 1)(\mathrm{H} 5)$ and also that

$$
\widehat{W}(\xi) \geq \max \left\{1, \frac{2}{N-1}\right\} \sum_{k=2}^{N}\left|\xi_{k} \partial_{k} \widehat{W}(\xi)\right|+\left|\xi_{1} \partial_{1} \widehat{W}(\xi)\right|, \text { for a.a. } \xi \in \mathbb{R}^{N} .
$$

Suppose that $c>c_{s}(W)$. Then nontrivial solutions of (NTWc) in $\mathcal{E}\left(\mathbb{R}^{N}\right)$ do not exist.

Corollary 1.5. Assume that $W$ satisfies $(\mathrm{H} 1)(\mathrm{H} 5)$, Suppose that

$$
c_{s}(W)<c \leq c_{s}(W)\left(1+\inf _{\xi \in \mathbb{R}^{N}} \frac{(N-1) \widehat{W}(\xi)}{\sum_{k=2}^{N}\left|\xi_{k} \partial_{k} \widehat{W}(\xi)\right|}\right)^{1 / 2} \text {, for a.a. } \xi \in \mathbb{R}^{N} .
$$

Then nontrivial solutions of (NTWc) in $\mathcal{E}\left(\mathbb{R}^{N}\right)$ do not exist.

Concerning the static waves, we have the following result.

Theorem 1.6. Assume that $W$ satisfies $(\mathrm{H} 1)(\mathrm{H} 4)$, Suppose that $c=0$ and that

$$
\xi_{j} \partial_{j} \widehat{W}(\xi) \leq 0 \text {, for a.a. } \xi \in \mathbb{R}^{N},
$$

for all $j \in\{1, \ldots, N\}$. Then nontrivial solutions of (NTWc) in $\mathcal{E}\left(\mathbb{R}^{N}\right)$ do not exist.

Note that in the case $W=a \delta, a>0, \widehat{W}=a$ and so that $\nabla \widehat{W}=0$. Then conditions (1.10), (1.11) and (1.12) hold. Therefore, invoking Corollary 1.4 or 1.5 and Theorem 1.6 we obtain the nonexistence of nontrivial solutions for all

$$
c \in\{0\} \cup(\sqrt{2 a}, \infty) .
$$


In particular, considering $a=1$, we recover Theorem 1.1] in the cases (i) and (ii)

So far, in view of (H5), we have assumed that $\widehat{W}$ is regular in a neighborhood of the origin, which in particular allows us to define $c_{s}(W)$. However there are interesting examples of kernels provided by the physical literature such that $\widehat{W}$ is not continuous at the origin and then $c_{s}(W)$ is not properly defined. For this reason we will work with a more general geometric condition on $\widehat{W}$. More precisely, denoting by $\left\{e_{k}\right\}_{k \in\{1, \ldots, N\}}$ the canonical unitary vectors of $\mathbb{R}^{N}$, we introduce the function

$$
w_{j}\left(\nu_{1}, \nu_{2}\right):=\widehat{W}\left(\nu_{1} e_{1}+\nu_{2} e_{j}\right), \quad\left(\nu_{1}, \nu_{2}\right) \in \mathbb{R}^{2}, j \in\{2, \ldots, N\}
$$

and the set

$$
\Gamma_{j, c}:=\left\{\nu=\left(\nu_{1}, \nu_{2}\right) \in \mathbb{R}^{2}:|\nu|^{4}+2 w_{j}(\nu)|\nu|^{2}-c^{2} \nu_{1}^{2}=0\right\} .
$$

Then Theorem 1.3 can be generalized if we replace (H5) by the condition

(H6) For all $j \in\{2, \ldots, N\}$ and $c>0$, there exist $\delta>0$ and two functions $\gamma_{j, c}^{+}$ and $\gamma_{j, c}^{-}$, defined on the interval $(0, \delta)$, such that the set $\Gamma_{j, c} \cap B(0, \delta)$ has Lebesgue measure zero, $\gamma_{j, c}^{ \pm} \in C^{1}((0, \delta))$, and

$$
\gamma_{j, c}^{+}(t)>0, \quad \gamma_{j, c}^{-}(t)<0, \quad\left(t, \gamma_{j, c}^{ \pm}(t)\right) \in \Gamma_{j, c}, \quad \text { for all } t \in(0, \delta) .
$$

Moreover, the following limits exist and are equal

$$
\lim _{t \rightarrow 0^{+}}\left(\frac{\gamma_{j, c}^{+}(t)}{t}\right)^{2}=\lim _{t \rightarrow 0^{+}}\left(\frac{\gamma_{j, c}^{-}(t)}{t}\right)^{2}=: \ell_{j, c} .
$$

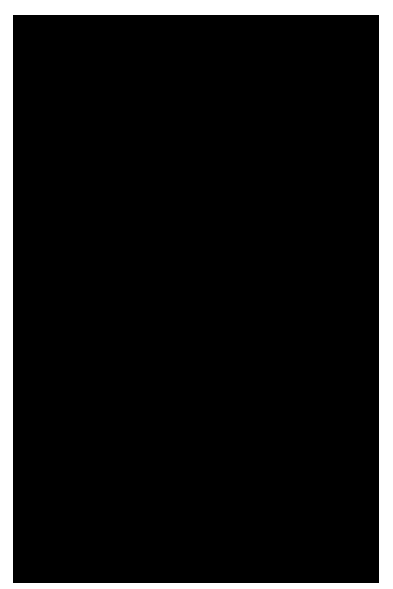

Figure 1. The curves $\gamma_{j, c}^{ \pm}$of condition (H6).

Figure 1 illustrates condition (H6) The fact that (H5) and (1.6) actually imply (H6) is proved in Section 4 (see Lemma 4.1). We also note that from (H6) we infer that $\lim _{t \rightarrow 0^{+}} \gamma_{j, c}^{ \pm}(t)=0$. Moreover, if $\widehat{W}$ is even in each component, that is

$$
\widehat{W}\left((-1)^{m_{1}} x_{1},(-1)^{m_{2}} x_{2}, \ldots,(-1)^{m_{N}} x_{N}\right)=\widehat{W}\left(x_{1}, x_{2}, \ldots, x_{N}\right),
$$

for all $\left(m_{1}, \ldots, m_{N}\right) \in\{0,1\}^{N}$, then $\gamma_{j, c}^{-}=-\gamma_{j, c}^{+}$, for all $j \in\{2, \ldots, N\}$. 
On the other hand, if the values $\ell_{j, c}$ are positive, a necessary condition for the existence of a nontrivial finite energy solution of (NTWC) is that they are equal.

Lemma 1.7. Let $c>0$. Assume that $W$ satisfies $(\mathrm{H} 1)(\mathrm{H} 4)$ and $(\mathrm{H} 6)$ with $\ell_{j, c}>0$, for all $j \in\{2, \ldots, N\}$. Let $v \in \mathcal{E}\left(\mathbb{R}^{N}\right)$ be a nontrivial solution of (NTWc) in $\mathcal{E}\left(\mathbb{R}^{N}\right)$. Then

$$
\ell_{1, c}=\ell_{2, c}=\cdots=\ell_{N, c}
$$

Now we are ready to state our main result in its general form.

Theorem 1.8. Let $c>0$. Assume that $W$ satisfies $(\mathrm{H} 1)(\mathrm{H} 4)$ and $(\mathrm{H} 6)$, with

$$
\ell_{c}:=\ell_{1, c}=\ell_{2, c}=\cdots=\ell_{N, c}>0 .
$$

Suppose that there exist constants $\sigma_{1}, \ldots, \sigma_{N} \in \mathbb{R}$ such that

$$
\widehat{W}(\xi)+\ell_{c} \sum_{k=2}^{N} \sigma_{k} \xi_{k} \partial_{k} \widehat{W}(\xi)-\sigma_{1} \xi_{1} \partial_{1} \widehat{W}(\xi) \geq 0, \text { for a.a. } \xi \in \mathbb{R}^{N},
$$

and

$$
\sum_{k=2}^{N} \sigma_{k}+\min \left\{-\sigma_{1}-1, \frac{\sigma_{1}-1}{\ell_{c}+2}, 2 \ell_{c} \sigma_{j}+\sigma_{1}-1\right\} \geq 0,
$$

for all $j \in\{2, \ldots, N\}$. Then nontrivial solutions of (ㅈc) in $\mathcal{E}\left(\mathbb{R}^{N}\right)$ do not exist.

Finally, we give the corresponding analogue of Corollaries 1.4 1.5.

Corollary 1.9. Let $c>0$. Assume that $W$ satisfies $(\mathrm{H} 1)(\mathrm{H} 4),(\mathrm{H} 6)$ and (1.15). Suppose that either (1.10) or

$$
l_{c} \leq \inf _{\xi \in \mathbb{R}^{N}} \frac{(N-1) \widehat{W}(\xi)}{\sum_{k=2}^{N}\left|\xi_{k} \partial_{k} \widehat{W}(\xi)\right|}
$$

hold. Then nontrivial solutions of (NTWC) in $\mathcal{E}\left(\mathbb{R}^{N}\right)$ do not exist.

1.5. Examples. In this subsection we provide some potentials of physical interest for which the Cauchy problem for (1.1) is globally well-posed (see [13]).

(I) Given the spherically symmetric interaction of particles, in physical models it is usual to suppose that $W$ is radial and then so is its Fourier transform, namely

$$
\widehat{W}(\xi)=\rho(|\xi|),
$$

for some function $\rho:[0, \infty) \rightarrow \mathbb{R}$. Assuming that $\rho$ is differentiable, we compute

$$
\xi_{k} \partial_{k} \widehat{W}(\xi)=\rho^{\prime}(|\xi|) \frac{\xi_{k}^{2}}{|\xi|}, \quad \text { for all } \xi \in \mathbb{R}^{N} \backslash\{0\} .
$$

Then, using that $\sum_{k=2}^{N} \xi_{k}^{2}=|\xi|^{2}-\xi_{1}^{2}$ and that $\left|\xi_{k}\right| \leq|\xi|$, we obtain that conditions (1.10) and (1.11) are respectively satisfied if

$$
\max \left\{1, \frac{2}{N-1}\right\} \leq \inf _{r>0} \frac{\rho(r)}{\left|\rho^{\prime}(r)\right| r},
$$


and

$$
2 \rho(0)<c^{2} \leq 2 \rho(0)\left(1+\inf _{r>0} \frac{\rho(r)}{\left|\rho^{\prime}(r)\right| r}\right) .
$$

We consider now a generalization of the model proposed by Shchesnovich and Kraenkel 29]

$$
\rho(r)=\frac{1}{\left(1+a r^{2}\right)^{b / 2}}, \quad a, b>0,
$$

so that

$$
c_{s}:=c_{s}(W)=\sqrt{2}
$$

It is immediate to verify that hypotheses (H1) (H3) (H5) are satisfied. Also, since $\widehat{W} \in L^{\infty}\left(\mathbb{R}^{N}\right),(\mathrm{H} 2)$ is fulfilled for $N=2,3$. Moreover, by Proposition 6.1.5 in [15, we conclude that $W \in L^{1}\left(\mathbb{R}^{N}\right) \cap L^{N}\left(\mathbb{R}^{N}\right)$ for $N \geq 4$ provided that $b>N-1$. On the other hand,

$$
\inf _{r>0} \frac{\rho(r)}{\left|\rho^{\prime}(r)\right| r}=\inf _{r>0} \frac{1+a r^{2}}{a b r^{2}}=\frac{1}{b} .
$$

Therefore, using (1.18)-(1.21) and invoking Corollaries 1.411.5 and Theorem 1.6 we conclude that in the following cases there is nonexistence of nontrivial solutions of (NTWc) in $\mathcal{E}\left(\mathbb{R}^{N}\right)$

(a) $N=2, b \leq 1 / 2, c \in\left(c_{s}, \infty\right)$.

(b) $N=2, b>1 / 2, c \in\left(c_{s}, \sqrt{2+2 / b}\right)$.

(c) $N=3, b \leq 1, c \in\left(c_{s}, \infty\right)$.

(d) $N=3, b>1, c \in\left(c_{s}, \sqrt{2+2 / b}\right)$.

(e) $N \geq 4, b>N-1, c \in\left(c_{s}, \sqrt{2+2 / b}\right)$.

(f) $N=2$ or $3, c=0$.

(g) $N \geq 4, b>N-1, c=0$.

We remark that if $b \rightarrow 0, \widehat{W} \rightarrow 1$ and then $W \rightarrow \delta$ in a distributional sense. Thus the cases (a) and (c) could be seen as a generalization of Theorem 1.1] in the cases (i) and (ii),

(II) Let $N=2,3$ and

$$
W_{\varepsilon}=\delta+\varepsilon f, \quad \varepsilon \geq 0,
$$

where $f$ is an even real-valued function, such that $f,|x|^{2} f,|x| \nabla f \in L^{1}\left(\mathbb{R}^{N}\right)$. Then $\widehat{W}_{\varepsilon}=1+\varepsilon \widehat{f} \in C^{2}\left(\mathbb{R}^{N}\right)$. Since

$$
\widehat{x_{j} \partial_{k} f}=-\left(\delta_{j, k} \widehat{f}+\xi_{k} \partial_{j} \widehat{f}\right),
$$

we have

$$
\|\widehat{f}\|_{L^{\infty}\left(\mathbb{R}^{N}\right)} \leq\|f\|_{L^{1}\left(\mathbb{R}^{N}\right)}, \quad\left\|\xi_{k} \partial_{j} \widehat{f}\right\|_{L^{\infty}\left(\mathbb{R}^{N}\right)} \leq\|f\|_{L^{1}\left(\mathbb{R}^{N}\right)}+\left\|x_{j} \partial_{k} f\right\|_{L^{1}\left(\mathbb{R}^{N}\right)} .
$$

Then we see that $W$ satisfies conditions (H1) (H5) provided that $\varepsilon<\|f\|_{L^{1}\left(\mathbb{R}^{N}\right)}^{-1}$ and that the sonic speed given by

$$
c_{s}:=c_{s}(W)=\left(2+2 \varepsilon \int_{\mathbb{R}^{N}} f\right)^{1 / 2},
$$


is well-defined. Moreover (1.10) is fulfilled if

$$
\varepsilon<\left(4\|f\|_{L^{1}\left(\mathbb{R}^{N}\right)}+\sum_{k=1}^{N}\left\|x_{k} \partial_{k} f\right\|_{L^{1}\left(\mathbb{R}^{N}\right)}\right)^{-1} .
$$

Therefore, under condition (1.23), Corollary 1.4 implies the nonexistence of nontrivial solutions of (NTWc) in $\mathcal{E}\left(\mathbb{R}^{N}\right)$ for any $c \in\left(c_{s}, \infty\right)$.

(III) The following potential used in [9, 30, to model dipolar forces in a quantum gas yields an example in $\mathbb{R}^{3}$ where the speed of sound is not properly defined. Let

$$
W=a \delta+b K, \quad a, b \in \mathbb{R},
$$

where $K$ is the singular kernel

$$
K(x)=\frac{x_{1}^{2}+x_{2}^{2}-2 x_{3}^{2}}{|x|^{5}}, \quad x \in \mathbb{R}^{3} \backslash\{0\} .
$$

In the sequel, we will deduce from Lemma 1.7 and Theorem 1.8 that there is nonexistence of nontrivial finite energy solutions of (NTWc) in $\mathcal{E}\left(\mathbb{R}^{N}\right)$ for all

$$
(2 \max \{a-\tilde{b}, a\})^{1 / 2}<c<\infty,
$$

with $\tilde{b}=(4 \pi b) / 3$, provided that $a>0$ and either

$$
a \geq \tilde{b} \geq 0 \quad \text { or } \quad a>-2 \tilde{b} \geq 0 .
$$

We now turn to the proof of condition (1.24). In fact, since (see [9])

$$
\widehat{W}(\xi)=a+\tilde{b}\left(\frac{3 \xi_{3}^{2}}{|\xi|^{2}}-1\right), \quad \xi \in \mathbb{R}^{3} \backslash\{0\},
$$

$W$ satisfies (H1) (H4) if one of the conditions in (1.25) holds. However, $\widehat{W}$ is not continuous at the origin. More precisely, in terms of the function defined in (1.14), we have that $w_{2}$ is constant equal to $a>0$ and by Lemma 4.1 there exist curves $\gamma_{2}^{ \pm}$with $\ell_{2, c}=c^{2} /(2 a)-1$. On the other hand, $w_{3}$ is not continuous at the origin but assuming (1.24) we can explicitly solve the algebraic equation

$$
\left(x^{2}+y^{2}\right)^{2}+2 w_{3}(x, y)\left(x^{2}+y^{2}\right)-c^{2} x^{2}=0
$$

and deduce that

$$
\gamma_{3, c}^{ \pm}(t)= \pm \sqrt{-t^{2}-a-2 \tilde{b}+\sqrt{6 \tilde{b} t^{2}+(a+2 \tilde{b})^{2}+c^{2} t^{2}}}
$$

for $|t|<c^{2}-2(a-\tilde{b})$. Therefore $(\mathrm{H} 6)$ holds and $\ell_{3, c}=-1+\left(6 \tilde{b}+c^{2}\right) /(2(a+2 \tilde{b}))$. Note that by (1.25), $\ell_{3, c}$ is a well-defined positive constant. By Lemma 1.7, a necessary condition so that the equation (NTWc) has nontrivial solutions is $\ell_{3, c}=\ell_{2, c}$, which leads us to

$$
\left(c^{2}-3 a\right) b=0 .
$$

The case $b=0$ has already been analyzed (see (1.13)). If $b \neq 0$, we obtain $c^{2}=3 a$. Hence $\ell_{c}:=\ell_{2, c}=\ell_{3, c}=1 / 2$. Then, taking $\sigma_{1}=0$ and $\sigma_{2}=\sigma_{3}=1 / 2$, (1.17) is satisfied and the l.h.s. of (1.16) reads

$$
a+\tilde{b}\left(3 \frac{\xi_{3}^{2}}{|\xi|^{2}}\left(1-\frac{\xi_{2}^{2}}{2|\xi|^{2}}\right)-1\right)+\frac{3 \tilde{b}}{2} \frac{\xi_{3}^{2}}{|\xi|^{2}}\left(1-\frac{\xi_{3}^{2}}{\left|\xi^{2}\right|}\right)
$$

which is nonnegative by (1.25). Therefore, by Theorem 1.8, there is nonexistence of nontrivial solutions of (1. 
As proved in [13, the Cauchy problem is also globally well-posed for other interactions such as the soft core potential

$$
W(x)=\left\{\begin{array}{lc}
1, & \text { if }|x|<a, \\
0, & \text { otherwise }
\end{array}\right.
$$

with $a>0$. However, our results do not apply to this kernel, since the changes of sign of $\widehat{W}$ will prevent that an inequality such as (1.16) can be satisfied. Moreover, in this case the energy could be negative making more difficult the analysis. Nevertheless, $\widehat{W}$ is positive near the origin and the sonic speed is still well defined, so that it is an open question to establish which are the exact implications of change of sign of the Fourier transform in the nonexistence results.

1.6. Outline of the proofs and organization of the paper. We recall that Theorem 1.1 (i) follows from a classical Pohozaev identity. Gravejat in [16 proves Theorem 1.1 (ii) by combining the respective Pohozaev identity with an integral equality obtained from the Fourier analysis of the equation satisfied by $1-|v|^{2}$. Our results are derived in the same spirit. In the next section we prove that conditions (H1) and (H2) imply the regularity of solutions of (NTWc). In Section 3 we prove that condition (H6) allows us to generalize the arguments in [16] so that we can derive the integral identity (3.1). The fact that the set $\Gamma_{j, c}$ is described by the curves $\gamma_{j, c}^{ \pm}$is a consequence of the Morse lemma, as explained in Section 4.

In Section 5 we establish a Pohozaev identity for (NTWc) with a "remainder term" depending on the derivatives of $\widehat{W}$. Although this identity can be formally obtained for rapidly decaying functions, its proof for functions in $\mathcal{E}\left(\mathbb{R}^{N}\right)$ is the major technical difficulty of this paper and relies on Fourier analysis and the fact that $W$ is even. As in [8, we then see in Section [6 that Theorem [1.6] is as straightforward consequence of this relation.

In Section 6 we also show that we can recast the identities described above as a suitable linear system of equations for which we can invoke the Farkas lemma to obtain the nonexistence conditions given in Theorems 1.8 and 1.3. The corollaries stated in Subsection 1.4 then follow by choosing the values of $\sigma_{1}, \ldots, \sigma_{N}$ appropriately.

Notations. We adopt the standard notation $C(\cdot, \cdot, \ldots)$ to represent a generic constant that depends only on each of its arguments. For any $x, y \in \mathbb{R}^{N}, z, w \in \mathbb{C}$, we denote the inner products in $\mathbb{R}^{N}$ and $\mathbb{C}$, respectively, by $x . y=\sum_{i=1}^{N} x_{i} y_{i}$ and $\langle z, w\rangle=\operatorname{Re}(z \bar{w})$. The Kronecker delta $\delta_{k, j}$ takes the value one if $k=j$ and zero otherwise. $\mathcal{F}(f)$ or $\widehat{f}$ stand for the Fourier transform of $f$, namely

$$
\mathcal{F}(f)(\xi)=\widehat{f}(\xi)=\int_{\mathbb{R}^{N}} f(x) e^{-i x \cdot \xi} d x,
$$

and $\mathcal{F}^{-1}$ for its inverse.

From now on we fix $c \geq 0$. We denote by $v=v_{1}+i v_{2}\left(v_{1}, v_{2}\right.$ real-valued) a solution of (NTWc) in $\mathcal{E}\left(\mathbb{R}^{N}\right)$. We also set the real-valued functions

$$
\rho:=|v|=\left(v_{1}^{2}+v_{2}^{2}\right)^{1 / 2}, \quad \eta:=1-|v|^{2} .
$$




\section{Regularity of SOlutions}

Lemma 2.1. Assume that $W \in \mathcal{M}_{2,2}\left(\mathbb{R}^{N}\right)$. Then $v \in W_{\mathrm{loc}}^{2,4 / 3}\left(\mathbb{R}^{N}\right)$. Suppose further that $2 \leq N \leq 3$. Then $v$ is smooth and bounded. Moreover, $\eta$ and $\nabla v$ belong to $W^{k, p}\left(\mathbb{R}^{N}\right)$, for all $k \in \mathbb{N}, 2 \leq p \leq \infty$.

Proof. Let $\bar{x} \in \mathbb{R}^{N}$ and $B_{r}:=B(\bar{x}, r)$ the ball of center $\bar{x}$ and radius $r$. Then

$$
\|v\|_{L^{4}\left(B_{1}\right)}=\left\||v|^{2}\right\|_{L^{2}\left(B_{1}\right)} \leq\left\||v|^{2}-1\right\|_{L^{2}\left(\mathbb{R}^{N}\right)}+\|1\|_{L^{2}\left(B_{1}\right)} \leq E(v)+C(N) .
$$

On the other hand, we can decompose $v$ as $v=z_{1}+z_{2}+z_{3}$, where $z_{1}, z_{2}$ and $z_{3}$ are the solutions of the following equations

$$
\begin{gathered}
\left\{\begin{array}{cl}
-\Delta z_{1}=0, & \text { in } B_{1}, \\
z_{1}=v, & \text { on } \partial B_{1},
\end{array}\right. \\
\left\{\begin{array}{cl}
-\Delta z_{2}=i c \partial_{1} v, & \text { in } B_{1}, \\
z_{2}=0, & \text { on } \partial B_{1},
\end{array}\right. \\
\left\{\begin{array}{cl}
-\Delta z_{3}=v(W * \eta), & \text { in } B_{1}, \\
z_{3}=0, & \text { on } \partial B_{1} .
\end{array}\right.
\end{gathered}
$$

Since $z_{1}$ is a harmonic function,

$$
\left\|z_{1}\right\|_{C^{k}\left(B_{1 / 2}\right)} \leq C(N, k, E(v)),
$$

for all $k \in \mathbb{N}$. Using the Hölder inequality, (2.1) and elliptic regularity estimates, we also have

$$
\left\|z_{2}\right\|_{W^{2,2}\left(B_{1}\right)} \leq C(N, E(v)), \quad\left\|z_{3}\right\|_{W^{2,4 / 3}\left(B_{1}\right)} \leq C(N, E(v))\|\widehat{W}\|_{L^{\infty}\left(\mathbb{R}^{N}\right)}\|\eta\|_{L^{2}\left(\mathbb{R}^{N}\right)} .
$$

Therefore $\|v\|_{W^{2,4 / 3\left(B_{1 / 2}\right)}} \leq C(N, E(v), \eta, W)$. Furthermore, by the Sobolev embedding theorem we deduce that $\|v\|_{L^{\infty}\left(B_{1 / 2}\right)}$ is bounded for $N=2$ and then this bound holds uniformly in $\mathbb{R}^{2}$. If $N=3$, we conclude that $\|v\|_{L^{12}\left(B_{1 / 2}\right)}$ is uniformly bounded. Then using the same decomposition (2.2)-(2.4) in the ball $B_{1 / 4}$, identical arguments prove that $\|v\|_{W^{2,12 / 7}\left(B_{1 / 4}\right)} \leq C(N, E(v), \eta, W)$, which by the Sobolev embedding theorem in dimension three implies that $\|v\|_{L^{\infty}\left(B_{1 / 4}\right)}$ is uniformly bounded. Consequently, $v \in L^{\infty}\left(\mathbb{R}^{N}\right)$ for $N=2,3$.

Finally, using again (2.2)-(2.4) and a standard bootstrap argument, we conclude that $v \in W^{k, \infty}\left(\mathbb{R}^{N}\right)$ for all $k \in \mathbb{N}$.

Now, setting $w=\partial_{j} v, j \in\{1, \ldots, N\}$, and differentiating (NTWc) with respect to $x_{j}$, we obtain for any $\lambda \in \mathbb{R}$

$$
L_{\lambda}(w):=-\Delta w-i c \partial_{1} w+\lambda w=\partial_{j} v(W * \eta)+v\left(W * \partial_{j} \eta\right)+\lambda w, \quad \text { in } \mathbb{R}^{N} .
$$

Since $\nabla v \in L^{\infty}\left(\mathbb{R}^{N}\right) \cap L^{2}\left(\mathbb{R}^{N}\right)$, we deduce that the r.h.s. belongs to $L^{2}\left(\mathbb{R}^{N}\right)$. Then, for $\lambda>0$ large enough, we can apply the Lax-Milgram theorem to the operator $L_{\lambda}$ to deduce that $w \in H^{2}\left(\mathbb{R}^{N}\right)$. Thus $\nabla v \in H^{2}\left(\mathbb{R}^{N}\right)$ and a bootstrap argument shows that $\nabla v \in H^{k}\left(\mathbb{R}^{N}\right)$, for all $k \in \mathbb{N}$ and therefore, by interpolation, $\nabla v, \eta \in W^{k, p}\left(\mathbb{R}^{N}\right)$, for all $p \geq 2$ and $k \in \mathbb{N}$. 
In Lemma 2.1. we needed to differentiate the equation (NTWc) to improve the regularity, which required that $W * \nabla \eta$ was well-defined. If $N \geq 4$, proceeding as in Lemma 2.1 we can only infer that $\nabla \eta \in L_{\text {loc }}^{4 / 3}\left(\mathbb{R}^{N}\right)$ so that it is not clear that we can give a sense to the term $W * \nabla \eta$. On the other hand, if $N \geq 3$, the fact that $\nabla v \in L^{2}\left(\mathbb{R}^{N}\right)$ implies that there exists $z_{0} \in \mathbb{C}$ with $\left|z_{0}\right|=1$ such that $v-z_{0} \in L^{\frac{2 N}{N-2}}\left(\mathbb{R}^{N}\right)$ (see e.g. [19, Theorem 4.5.9]). Moreover, since (NTWc) is invariant by a change of phase, we can assume that $v-1 \in L^{\frac{2 N}{N-2}}\left(\mathbb{R}^{N}\right)$. Therefore,

$$
\nabla \eta=-2\langle v-1, \nabla v\rangle-2\langle 1, \nabla v\rangle \in L^{N /(N-1)}\left(\mathbb{R}^{N}\right)+L^{2}\left(\mathbb{R}^{N}\right) .
$$

Then it would be reasonable to suppose that $W \in \mathcal{M}_{N / N-1, q}\left(\mathbb{R}^{N}\right)$, for some $q \geq$ $N / N-1$. However, this is not enough to invoke the elliptic regularity estimates and that is reason why we work with the assumption (1.7) in (H2) if $N \geq 4$. We remark that to establish precise conditions on $W$ that ensure the regularity of solutions of (NTWC) in higher dimensions goes beyond the scope of this paper.

Lemma 2.2. Let $N \geq 4$. Assume that $W$ satisfies $(\mathrm{H} 2)$, Then $v$ is bounded and smooth. Moreover, $\eta$ and $\nabla v$ belong to $W^{k, p}\left(\mathbb{R}^{N}\right)$, for all $k \in \mathbb{N}, 2 \leq p \leq \infty$.

Proof. From (1.7), by duality (see e.g. [15]) we infer that $W \in \mathcal{M}_{1, N}\left(\mathbb{R}^{N}\right) \cap$ $\mathcal{M}_{1,2 N /(N+2)}\left(\mathbb{R}^{N}\right)$. Then, from the Riesz-Thorin interpolation theorem and the fact that $(1 / 2,(N-2) /(2 N))$ and $((N-1) / N,(N-2) /(2 N))$ belong to the convex hull of

$$
\left\{\left(\frac{1}{2}, \frac{1}{2}\right),\left(\frac{N-1}{N}, 0\right),\left(\frac{N-2}{2 N}, 0\right),\left(1, \frac{1}{N}\right),\left(1, \frac{N+2}{2 N}\right)\right\},
$$

we conclude that

$$
W \in \mathcal{M}_{2,2 N /(N-2)}\left(\mathbb{R}^{N}\right) \text { and } W \in \mathcal{M}_{N /(N-1), 2 N /(N-2)}\left(\mathbb{R}^{N}\right) .
$$

As mentioned before, we can assume that $\tilde{v}:=v-1 \in L^{\frac{2 N}{N-2}}\left(\mathbb{R}^{N}\right)$. Then using (H2), (2.5) and (2.6), we are led to

$$
W * \eta, W * \nabla \eta \in L^{\infty}\left(\mathbb{R}^{N}\right) \cap L^{2 N /(N-2)}\left(\mathbb{R}^{N}\right) .
$$

Now we recast (NTWC as

$$
L_{\lambda}(\tilde{v}):=-\Delta \tilde{v}-i c \partial_{1} \tilde{v}+\lambda \tilde{v}=\tilde{v}((W * \eta)+\lambda)+W * \eta, \quad \text { in } \mathbb{R}^{N},
$$

for some $\lambda>0$. By (2.7), the r.h.s. of (2.8) belongs to $L^{2 N /(N-2)}\left(\mathbb{R}^{N}\right)$. Then choosing $\lambda$ large enough, we can apply elliptic regularity estimates to the operator $L_{\lambda}$ to conclude that $\tilde{v} \in W^{2,2 N /(N-2)}\left(\mathbb{R}^{N}\right)$. Then

$\partial_{j, k} \eta=-2\left(\left\langle v-1, \partial_{j, k} v\right\rangle+\left\langle\partial_{j} v, \partial_{k} v\right\rangle+\left\langle 1, \partial_{j, k} v\right\rangle\right) \in L^{N /(N-1)}\left(\mathbb{R}^{N}\right)+L^{2 N /(N-2)}\left(\mathbb{R}^{N}\right)$, for any $1 \leq j, k \leq N$. Therefore, by (1.7) and (2.6), $W * \partial_{j, k} \eta \in L^{\infty}\left(\mathbb{R}^{N}\right) \cap$ $L^{2 N /(N-2)}$. Thus the r.h.s. of $(2.8)$ belongs to $W^{2,2 N /(N-2)}\left(\mathbb{R}^{N}\right)$, so that $\tilde{v} \in$ $W^{4,2 N /(N-2)}\left(\mathbb{R}^{N}\right)$. A bootstrap argument yields that $\tilde{v} \in W^{k, 2 N /(N-2)}\left(\mathbb{R}^{N}\right)$, for any $k \in \mathbb{N}$. By the Sobolev embedding theorem, we conclude that $v \in W^{k, \infty}\left(\mathbb{R}^{N}\right)$ for any $k \in \mathbb{N}$. Then the conclusion follows as in Lemma 2.1

Lemma 2.3. Let $W \in L^{1}\left(\mathbb{R}^{N}\right)$ if $2 \leq N \leq 3$ and $W \in L^{1}\left(\mathbb{R}^{N}\right) \cap L^{N}\left(\mathbb{R}^{N}\right)$ if $N \geq 4$. Then $W$ fulfills $(\mathrm{H} 2)$. 
Proof. Since $W \in L^{1}\left(\mathbb{R}^{N}\right)$, by the Young inequality we have

$$
\|W * f\|_{L^{p}\left(\mathbb{R}^{N}\right)} \leq\|W\|_{L^{1}\left(\mathbb{R}^{N}\right)}\|f\|_{L^{p}\left(\mathbb{R}^{N}\right)}, \quad \text { for any } p \in[1, \infty] .
$$

Then, taking $p=2$, we conclude that (H2) holds for $2 \leq N \leq 3$. For $N \geq 4$, we have $W \in L^{1}\left(\mathbb{R}^{N}\right) \cap L^{N}\left(\mathbb{R}^{N}\right)$. In particular, $W \in L^{2 N /(N+2)}\left(\mathbb{R}^{N}\right)$ and the Young inequality implies that

$$
\begin{aligned}
& \|W * f\|_{L^{\infty}\left(\mathbb{R}^{N}\right)} \leq\|W\|_{L^{N}\left(\mathbb{R}^{N}\right)}\|f\|_{L^{N /(N-1)\left(\mathbb{R}^{N}\right)}}, \\
& \|W * f\|_{L^{\infty}\left(\mathbb{R}^{N}\right)} \leq\|W\|_{L^{2 N /(N+2)\left(\mathbb{R}^{N}\right)}}\|f\|_{L^{2 N /(N-2)\left(\mathbb{R}^{N}\right)} .}
\end{aligned}
$$

Therefore (H2) is satisfied.

Corollary 2.4. Assume that $W$ satisfies $(\mathrm{H} 2)$. Then $v$ is smooth and bounded. Moreover, $\eta$ and $\nabla v$ belong to $W^{k, p}\left(\mathbb{R}^{N}\right)$, for all $k \in \mathbb{N}, 2 \leq p \leq \infty$, and

$$
\rho(x) \rightarrow 1, \nabla v(x) \rightarrow 0, \quad \text { as }|x| \rightarrow \infty .
$$

Furthermore, there exists a smooth lifting of $v$. More precisely, there exist $R_{0}>0$ and a smooth real-valued function $\theta$ defined on $B\left(0, R_{0}\right)^{c}$, with $\nabla \theta \in W^{k, p}\left(B\left(0, R_{0}\right)^{c}\right)$, for all $k \in \mathbb{N}, 2 \leq p \leq \infty$, such that

$$
\rho \geq \frac{1}{2} \quad \text { and } \quad v=\rho e^{i \theta} \quad \text { on } B\left(0, R_{0}\right)^{c} .
$$

Proof. The first part is exactly Lemmas 2.1 and 2.3. In particular, $v$ and $\nabla v$ are uniformly continuous on $\mathbb{R}^{N}$. Then, since $1-|v|^{2} \in L^{2}\left(\mathbb{R}^{N}\right)$ and $\nabla v \in L^{2}\left(\mathbb{R}^{N}\right)$, we obtain (2.9). The existence of the lifting satisfying (2.10) follows as in [25, Proposition 2.5]. From (2.10) we also deduce that

$$
|\nabla v|^{2}=|\nabla \rho|^{2}+\rho^{2}|\nabla \theta|^{2} \quad \text { on } B\left(0, R_{0}\right)^{c} .
$$

Since $\rho \geq 1 / 2$ on $B\left(0, R_{0}\right)^{c}$, we infer that $\nabla \theta \in W^{k, p}\left(B\left(0, R_{0}\right)^{c}\right)$, for all $k \in \mathbb{N}$, $2 \leq p \leq \infty$.

In virtue of Corollary 2.4, we introduce the function $\phi \in C^{\infty}\left(\mathbb{R}^{N}\right),|\phi| \leq 1$, such that $\phi=0$ on $B\left(0,2 R_{0}\right)$ and $\phi=1$ on $B\left(0,3 R_{0}\right)^{c}$. In this way, we can assume the function $\phi \theta$ is well-defined on $\mathbb{R}^{N}$. This will be useful in the next section to work with global functions in terms of $\theta$. In fact, we end this section with the following result.

Lemma 2.5. Assume that $W$ satisfies (H2), Then

$$
G:=v_{1} \nabla v_{2}-v_{2} \nabla v_{1}-\nabla(\phi \theta), \quad \text { on } \mathbb{R}^{N},
$$

belongs to $W^{k, p}\left(\mathbb{R}^{N}\right)$, for all $k \in \mathbb{N}$ and $1 \leq p \leq \infty$.

Proof. By Corollary 2.4, $G \in C^{\infty}\left(\mathbb{R}^{N}\right)$ and moreover

$$
G=-\eta \nabla \theta \quad \text { on } B\left(0,3 R_{0}\right)^{c} .
$$

Since $\nabla \theta \in W^{k, p}\left(B\left(0, R_{0}\right)^{c}\right)$ and $\eta \in W^{k, p}\left(B\left(0, R_{0}\right)^{c}\right)$, for all $k \in \mathbb{N}, 2 \leq p \leq \infty$, the conclusion follows. 


\section{AN INTEGRAL IDENTITY}

The aim of this section is to prove the following integral identity.

Proposition 3.1. Let $c>0$. Suppose that $(\mathrm{H} 2)$ and $(\mathrm{H} 6)$ hold with $\ell_{j, c}>0$, for some $j \in\{2, \ldots, N\}$. Then

$$
\int_{\mathbb{R}^{N}}\left(|\nabla v|^{2}+\eta(W * \eta)\right)=-c \frac{\ell_{j, c}}{1+\ell_{j, c}} \int_{\mathbb{R}^{N}}\left(v_{1} \partial_{1} v_{2}-v_{2} \partial_{1} v_{1}-\partial_{1}(\phi \theta)\right) .
$$

We note that since $W$ satisfies (H2), all the results of Section 2 hold. On the other hand, from (NTWc) we deduce that $\eta=1-|v|^{2}$ satisfies

$$
\Delta \eta=-F+2 W * \eta-2 c \partial_{1}(\phi \theta) .
$$

where

$$
F:=2|\nabla v|^{2}+2 \eta(W * \eta)+2 c G_{1} .
$$

and $G=\left(G_{1}, \ldots, G_{N}\right)$ was defined in (2.11). Considering real and imaginary parts in (NTWC) and multiplying them by $v_{2}$ and $v_{1}$, respectively, it follows that

$$
\operatorname{div}(G)=v_{1} \Delta v_{2}-v_{2} \Delta v_{1}-\Delta(\phi \theta)=\frac{c}{2} \partial_{1} \eta-\Delta(\phi \theta) .
$$

Therefore, from (3.2) and (3.3), we conclude that

$$
\Delta^{2} \eta-2 \Delta(W * \eta)+c^{2} \partial_{11}^{2} \eta=-\Delta F+2 c \partial_{1}(\operatorname{div} G), \quad \text { in } \mathbb{R}^{N} .
$$

Since we are assuming $(\mathrm{H} 2)$, by Corollary 2.4 and Lemma 2.5. we have that $F, G \in$ $W^{k, 1}\left(\mathbb{R}^{N}\right) \cap W^{k, 2}\left(\mathbb{R}^{N}\right)$, for all $k \in \mathbb{N}$, so that (3.4) stands in $L^{2}\left(\mathbb{R}^{N}\right)$. Taking the Fourier transform in equation (3.4) and setting

$$
R(\xi):=|\xi|^{4}+2 \widehat{W}(\xi)|\xi|^{2}-c^{2} \xi_{1}^{2} \quad \text { and } \quad H(\xi):=|\xi|^{2} \widehat{F}(\xi)-2 c \sum_{j=1}^{N} \xi_{1} \xi_{j} \widehat{G}_{j}(\xi)
$$

we get

$$
R(\xi) \widehat{\eta}(\xi)=H(\xi), \quad \text { in } L^{2}\left(\mathbb{R}^{N}\right) .
$$

Lemma 3.2. Let $c>0$. Suppose that (H2) and (H6) hold. Then for all $j \in$ $\{2, \ldots, N\}$,

$$
H\left(t e_{1}+\gamma_{j, c}^{ \pm}(t) e_{j}\right)=0, \quad \text { for all } t \in(0, \delta),
$$

where $\delta$ is given by $(\mathrm{H} 6)$.

Proof. We fix $j \in\{2, \ldots, N\}$ and we prove (3.6) for $\gamma_{j, c}^{+}$, since the proof for $\gamma_{j, c}^{-}$is analogous. To simplify the notation, we put $\gamma:=\gamma_{j, c}^{+}$. As stated before, $F, G \in$ $W^{k, 1}\left(\mathbb{R}^{N}\right) \cap W^{k, 2}\left(\mathbb{R}^{N}\right)$, for all $k \in \mathbb{N}$. In particular $F, G \in L^{1}\left(\mathbb{R}^{N}\right)$, so that $\widehat{F}$, $\widehat{G} \in C\left(\mathbb{R}^{N}\right)$. Thus $H$ is a continuous function on $\mathbb{R}^{N}$.

Let $\delta>0$ given by $(\mathrm{H} 6)]$ Arguing by contradiction, we suppose that there exist $t_{0} \in(0, \delta)$ and a constant $A>0$ such that $|H(\tilde{\xi})| \geq A$, where $\tilde{\xi}=t_{0} e_{1}+\gamma\left(t_{0}\right) e_{j}$. By the continuity of $H$, there exists $r>0$ such that $|H(\xi)| \geq A$, for all $\xi \in V_{r}$, where

$$
V_{r}=B(\tilde{\xi}, r) \cap\left\{\alpha e_{1}+\beta e_{j}: \alpha, \beta \in \mathbb{R}\right\} .
$$


Thus $V_{r}$ is a two-dimensional set and since $t_{0}>0$, we can choose $r$ small enough such that $0 \notin V_{r}$. Then (3.5) yields

$$
|\widehat{\eta}(\xi)|^{2} \geq \frac{A^{2}}{(R(\xi))^{2}}, \quad \text { for all } \xi \in V_{r} \backslash \Gamma_{j, c} .
$$

We claim that

$$
I:=\int_{V_{r} \backslash \Gamma_{j, c}} \frac{d \xi_{1} d \xi_{j}}{(R(\xi))^{2}}=+\infty .
$$

Since by hypothesis $\Gamma_{j, c} \cap B(0, \delta)$ has measure zero, (3.7) and (3.8) contradict that $\widehat{\eta} \in L^{2}\left(\mathbb{R}^{N}\right)$.

To prove (3.8), since $V_{r}$ is a two-dimensional set, we identify it as a subset of $\mathbb{R}^{2}$ and so that we write $e_{2}$ instead of $e_{j}$. Then, since $\Gamma_{j, c} \cap B(0, \delta)$ has measure zero,

$$
I=\int_{V_{r}} \frac{d \xi_{1} d \xi_{2}}{(R(\xi))^{2}}
$$

To compute the integral we "straighten out" the curve $\gamma$. Namely, we introduce the change of variables

$$
\begin{aligned}
& \xi_{1}=\nu_{1}=: \Phi_{1}\left(\nu_{1}, \nu_{2}\right), \\
& \xi_{2}=\nu_{2}+\gamma\left(\nu_{1}\right)=: \Phi_{2}\left(\nu_{1}, \nu_{2}\right) .
\end{aligned}
$$

Since $\gamma$ is a $C^{1}$-function, so is $\Phi$. Moreover, there is some set $U_{r}$ such that $V_{r}=$ $\Phi\left(U_{r}\right)$ and $|\operatorname{det}(J \Phi(\nu))|=1$ for all $\nu \in U_{r}$. Setting $F(\nu):=R(\Phi(\nu)), \nu \in U_{r}$, the change of variables theorem yields

$$
I=\int_{U_{r}} \frac{d \nu_{1} d \nu_{2}}{(F(\nu))^{2}}
$$

Furthermore, since $F \in C^{1}\left(U_{r}\right)$ and $F\left(\nu_{1}, 0\right)=0$ for all $\left(\nu_{1}, 0\right) \in U_{r}$, the Taylor theorem implies that for any $\left(\nu_{1}, \nu_{2}\right) \in U_{r}$, there is some $\bar{\nu} \in U_{r}$ such that

$$
F\left(\nu_{1}, \nu_{2}\right)=F\left(\nu_{1}, 0\right)+\frac{\partial F}{\partial \nu_{2}}(\bar{\nu}) \nu_{2}=\frac{\partial F}{\partial \nu_{2}}(\bar{\nu}) \nu_{2},
$$

On the other hand, by (H2), $\widehat{W} \in L^{\infty}\left(\mathbb{R}^{N}\right)$ and by (H3)] $\nabla W \in L^{\infty}\left(V_{r}\right)$, so that $\|\widehat{W}\|_{W^{1, \infty}\left(V_{r}\right)}<\infty$. Thus $\|\nabla F\|_{L^{\infty}\left(U_{r}\right)} \leq C(r, \gamma)\left(1+\|\widehat{W}\|_{W^{1, \infty}\left(V_{r}\right)}\right)$ and from (3.10) we conclude that

$$
|F(\nu)| \leq C(r, \gamma)\left(1+\|\widehat{W}\|_{W^{1, \infty}\left(V_{r}\right)}\right)\left|\nu_{2}\right|, \quad \text { for all } \nu \in U_{r} .
$$

From (3.9) and (3.11), taking $\tilde{\nu}=\left(\tilde{\nu}_{1}, \tilde{\nu}_{2}\right) \in U_{r}$ such that $\tilde{\xi}=\Phi(\tilde{\nu})$ and $\varepsilon>0$ small enough, we conclude that

$$
I \geq C(r, \gamma, \widehat{W}) \int_{U_{r}} \frac{d \nu_{1} d \nu_{2}}{\nu_{2}^{2}} \geq C(r, \gamma, \widehat{W}) \int_{\tilde{\nu}_{1}-\varepsilon}^{\tilde{\nu}_{1}+\varepsilon} \int_{-\varepsilon}^{\varepsilon} \frac{d \nu_{2} d \nu_{1}}{\nu_{2}^{2}}=+\infty,
$$

which concludes the proof.

Finally, we give the proof of identity (3.1).

Proof of Proposition 3.1. By Lemma 3.2, setting $\xi^{ \pm}(t)=t e_{1}+\gamma_{j, c}^{ \pm}(t) e_{j}$, we have

$$
\left(t^{2}+\left(\gamma_{j, c}^{ \pm}(t)\right)^{2}\right) \widehat{F}\left(\xi^{ \pm}(t)\right)-2 c t^{2} \widehat{G}_{1}\left(\xi^{ \pm}(t)\right)-2 c t \gamma_{j, c}^{ \pm}(t) \widehat{G}_{j}\left(\xi^{ \pm}(t)\right)=0, \quad t \in(0, \delta) .
$$


Dividing by $t^{2}$ and passing to the limit $t \rightarrow 0^{+}$,

$\left(1+\ell_{j, c}\right) \widehat{F}(0)-2 c \widehat{G}_{1}(0)-2 c \sqrt{\ell_{j, c}} \widehat{G}_{j}(0)=\left(1+\ell_{j, c}\right) \widehat{F}(0)-2 c \widehat{G}_{1}(0)+2 c \sqrt{\ell_{j, c}} \widehat{G}_{j}(0)=0$.

Therefore, since $\ell_{j, c}>0, \widehat{G}_{j}(0)=0$ and $\left(1+\ell_{j, c}\right) \widehat{F}(0)=2 c \widehat{G}_{1}(0)$, which is precisely (3.1).

As a consequence of Proposition 3.1, we obtain Lemma 1.7

Proof of Lemma 1.7. From (3.1), setting

$$
J(v)=\int_{\mathbb{R}^{N}}\left(|\nabla v|^{2}+\eta(W * \eta)\right) \text { and } P(v)=\int_{\mathbb{R}^{N}}\left(v_{1} \partial_{1} v_{2}-v_{2} \partial_{1} v_{1}-\partial_{1}(\phi \theta)\right),
$$

we infer that

$$
\ell_{j, c}(J(v)+c P(v))=-J(v) .
$$

Since $v$ is nonconstant and $\widehat{W} \geq 0$, we have that $J(v)>0$. Then we deduce from (3.12) that $J(v)+c P(v) \neq 0$ and

$$
\ell_{j, c}=-\frac{J(v)}{J(v)+c P(v)} .
$$

Since the r.h.s. of the equality does not depend on $j$, the conclusion follows.

\section{The Set $\Gamma_{j, c}$ Under the condition (H5)}

In Section 3 we have seen that identity (3.1) is a consequence of the structure of the set $\Gamma_{j, c}$. More precisely, it relies on the fact that $(\mathrm{H} 6)$ provides the existence of $\delta>0$ and two curves $\gamma_{j, c}^{ \pm}$such that

$$
\left\{\left(t, y^{ \pm}(t)\right): t \in(-\delta, \delta)\right\} \subseteq \Gamma_{j, c} .
$$

If $\widehat{W}$ is of class $C^{2}$ in a neighborhood of the origin and

$$
\alpha_{c}:=\frac{c^{2}}{\left(c_{s}(W)\right)^{2}}-1>0,
$$

we can use the Morse lemma to justify the existence of the curves $\gamma_{j, c}^{ \pm}$and to conclude that set $\Gamma_{j, c}$ consists of exactly these two curves near the origin. Therefore the set $\Gamma_{j, c}$ looks like Figure 2 and condition $(\mathrm{H} 6)$ is fulfilled.

Lemma 4.1. Assume that $(\mathrm{H} 1)$ and $(\mathrm{H} 5)$ hold. Assume also that $\alpha_{c}>0$. Then, for each $j \in\{2, \ldots, N\}$, there exist $\delta>0$ and functions $y^{ \pm} \in C^{1}((-\delta, \delta)) \cap C^{2}((-\delta, \delta) \backslash$ $\{0\})$ such that

$$
\Gamma_{j, c} \cap B(0, \delta)=\left\{\left(t, y^{ \pm}(t)\right): t \in(-\delta, \delta)\right\}
$$

Moreover,

$$
\lim _{t \rightarrow 0^{+}} y^{ \pm}(t) / t= \pm \sqrt{\alpha_{c}},
$$

$y^{+}$is strictly increasing and $y^{-}$is strictly decreasing. In particular, (H6) is satisfied with $l_{j, c}=\alpha_{c}$. 


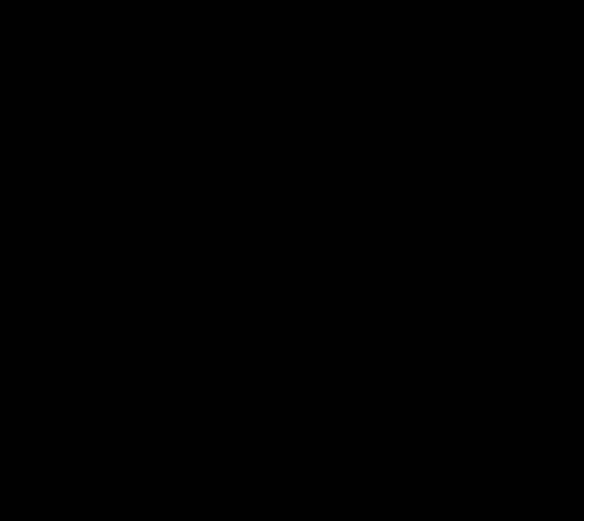

Figure 2. The set $\Gamma_{j, c}$ near the origin for $\widehat{W}$ of class $C^{2}$.

Proof. Let us set

$$
R_{j}(\nu):=|\nu|^{4}+2 w_{j}(\nu)|\nu|^{2}-c^{2} \nu_{1}^{2}, \quad \nu=\left(\nu_{1}, \nu_{2}\right) \in \mathbb{R}^{2} .
$$

In view of (H5) $R_{j} \in C^{2}\left(B\left(0, \delta_{0}\right)\right)$, for some $\delta_{0}>0$. Since $w_{j}$ is even, we have that $\partial_{1} w_{j}(0,0)=\partial_{2} w_{j}(0,0)=0$. Then we obtain $R_{j}(0,0)=0, \nabla R_{j}(0,0)=0$, $(4.3)$

$$
\frac{\partial^{2} R_{j}}{\partial \nu_{1}^{2}}(0,0)=-4 \alpha_{c} w_{j}(0,0)<0, \quad \frac{\partial^{2} R_{j}}{\partial \nu_{2}^{2}}(0,0)=4 w_{j}(0,0)>0, \quad \frac{\partial^{2} R_{j}}{\partial \nu_{1} \partial \nu_{2}}(0,0)=0 .
$$

Therefore by the Morse lemma (see e.g. [26, Theorem II]) there exist two neighborhoods of the origin $U, V \subset \mathbb{R}^{2}$ and a local diffeomorphism $\Phi: U \rightarrow V$ such that

$$
R_{j}\left(\Phi^{-1}(z)\right)=-2 \alpha_{c} w_{j}(0,0) z_{1}^{2}+2 w_{j}(0,0) z_{2}^{2}, \quad \text { for all } z=\left(z_{1}, z_{2}\right) \in V .
$$

Moreover, denoting $\Phi=\left(\Phi_{1}, \Phi_{2}\right)$ we have for $1 \leq j, k \leq 2$

$$
\frac{\partial \Phi_{j}}{\partial \nu_{k}}(\nu) \rightarrow \delta_{j, k}, \quad \text { as }|\nu| \rightarrow 0 .
$$

From (4.4) we deduce that near the origin the set of solutions of $R_{j}=0$ is given by the lines

$$
\left\{\left(t, \pm \sqrt{\alpha_{c}} t\right): t \in(-\delta, \delta)\right\}
$$

where we take $\delta>0$ such that the set is contained in $V$. Since $\Phi$ is a diffeomorphism we conclude that

$$
\Gamma_{j, c} \cap B(0, \delta)=\left\{\left(x_{1}^{ \pm}(t), x_{2}^{ \pm}(t)\right): t \in(-\delta, \delta)\right\},
$$

where

$$
\begin{aligned}
& \Phi_{1}\left(x_{1}^{ \pm}, x_{2}^{ \pm}\right)=t, \\
& \Phi_{2}\left(x_{1}^{ \pm}, x_{2}^{ \pm}\right)= \pm \sqrt{\alpha_{c}} t .
\end{aligned}
$$

Moreover, differentiating relation (4.7) with respect to $t$ and using (4.5), we infer that $\left(x_{1}^{ \pm}\right)^{\prime}(t) \rightarrow 1$ as $t \rightarrow 0$. Therefore we can recast (4.6) as in (4.1) with $y^{ \pm} \in$ 
$C^{1}((-\delta, \delta)) \cap C^{2}((-\delta, \delta) \backslash\{0\})$. Furthermore, differentiating (4.8) and using again (4.5) we conclude that

$$
\left(y^{ \pm}\right)^{\prime}(0)= \pm \sqrt{\alpha_{c}} .
$$

Since $y^{ \pm} \in C^{1}((-\delta, \delta))$, taking a possible smaller value $\delta$, this implies (4.2) and that $y^{+}$and $y^{-}$are strictly increasing and decreasing on $(-\delta, \delta)$, respectively.

\section{A Pohozaev identity}

In this section we establish the following Pohozaev identity.

Proposition 5.1. Assume that (H1) (H3) hold. Then

$E(v)=\int_{\mathbb{R}^{N}}\left|\partial_{1} v\right|^{2}+\frac{1}{4(2 \pi)^{N}} \int_{\mathbb{R}^{N}} \xi_{1} \partial_{1} \widehat{W}|\widehat{\eta}|^{2} d \xi$,

$E(v)=\int_{\mathbb{R}^{N}}\left|\partial_{j} v\right|^{2}-\frac{c}{2} \int_{\mathbb{R}^{N}}\left(v_{1} \partial_{1} v_{2}-v_{2} \partial_{1} v_{1}-\partial_{1}(\phi \theta)\right)+\frac{1}{4(2 \pi)^{N}} \int_{\mathbb{R}^{N}} \xi_{j} \partial_{j} \widehat{W}|\widehat{\eta}|^{2} d \xi$, for all $j \in\{2, \ldots, N\}$.

Note that by Lemma 2.5, $G_{1}=v_{1} \partial_{1} v_{2}-v_{2} \partial_{1} v_{1}-\partial_{1}(\phi \theta) \in L^{1}\left(\mathbb{R}^{N}\right)$, thus every integral in (5.1) and (5.2) is finite. As mentioned in Section 1, in the case that $W$ is the Dirac delta function this result is well-known (see [8, 6, 16, 25]). The standard technique is to introduce a function $\chi \in C^{\infty}(\mathbb{R})$, with $\chi(x)=1$ for $|x|<1$ and $\chi(x)=0$ for $|x|>2$, and $\chi_{n}(x):=\chi(x / n)$. Then, multiplying (NTWc) by $x_{j} \chi_{n} \partial_{j} \bar{v}$ and taking real part, we are led to

$$
\left\langle i c \partial_{1} v+\Delta v, x_{j} \chi_{n} \partial_{j} v\right\rangle-\frac{1}{2}(W * \eta) x_{j} \chi_{n} \partial_{j} \eta=0, \quad \text { on } \mathbb{R}^{N},
$$

where we have used that

$$
\left\langle v, \partial_{j} v\right\rangle=-\frac{1}{2} \partial_{j} \eta
$$

Concerning (5.3), we recall the following result.

Lemma $5.2\left(\left[8,6\right.\right.$, 16, 25]). Let $\varphi=\varphi_{1}+\varphi_{2} \in \mathcal{E}\left(\mathbb{R}^{N}\right) \cap C^{\infty}\left(\mathbb{R}^{N}\right)$. Assume that there exist $R^{*}>0$ and a smooth real-valued function $\tilde{\theta}$ defined on $B\left(0, R^{*}\right)^{c}$, with $\nabla \tilde{\theta} \in L^{2}\left(B\left(0, R^{*}\right)^{c}\right)$, such that

$$
|\varphi| \geq \frac{1}{2} \quad \text { and } \quad \varphi=|\varphi| e^{i \tilde{\theta}} \quad \text { on } B\left(0, R_{0}\right)^{c} .
$$

Let $\tilde{\phi} \in C^{\infty}\left(\mathbb{R}^{N}\right)$, such that $\tilde{\phi}=0$ on $B\left(0,2 R^{*}\right)$ and $\tilde{\phi}=1$ on $B\left(0,3 R^{*}\right)^{c}$. Then for all $j \in\{1, \ldots, N\}$, we have

$$
\begin{aligned}
& \lim _{n \rightarrow \infty} \int_{\mathbb{R}^{N}}\left\langle i \partial_{1} \varphi, x_{j} \chi_{n} \partial_{j} \varphi\right\rangle=\frac{1}{2}\left(1-\delta_{1, j}\right) \int_{\mathbb{R}^{N}}\left(\varphi_{1} \partial_{1} \varphi_{2}-\varphi_{2} \partial_{1} \varphi_{1}-\partial_{1}(\tilde{\phi} \tilde{\theta})\right), \\
& \lim _{n \rightarrow \infty} \int_{\mathbb{R}^{N}}\left\langle\Delta \varphi, x_{j} \chi_{n} \partial_{j} \varphi\right\rangle=-\int_{\mathbb{R}^{N}}\left|\partial_{j} \varphi\right|^{2}+\frac{1}{2} \int_{\mathbb{R}^{N}}|\nabla \varphi|^{2}, \\
& \lim _{n \rightarrow \infty}-\frac{1}{2} \int_{\mathbb{R}^{N}} x_{j} \chi_{n}\left(1-|\varphi|^{2}\right) \partial_{j}\left(1-|\varphi|^{2}\right)=\frac{1}{4} \int_{\mathbb{R}^{N}}\left(1-|\varphi|^{2}\right)^{2} .
\end{aligned}
$$


Therefore, from (5.3) and Lemma 5.2 Proposition 5.1 follows in the case $W=\delta$. To motivate our approach, let us briefly recall the proof of (5.6). First, we integrate by parts to obtain

$$
\begin{aligned}
A_{n}: & =-\frac{1}{2} \int_{\mathbb{R}^{N}} x_{j} \chi_{n}\left(1-|\varphi|^{2}\right) \partial_{j}\left(1-|\varphi|^{2}\right) \\
& =\frac{1}{2} \int_{\mathbb{R}^{N}} \chi_{n}\left(1-|\varphi|^{2}\right)^{2}+\frac{1}{2} \int_{\mathbb{R}^{N}} x_{j} \partial_{j} \chi_{n}\left(1-|\varphi|^{2}\right) \partial_{j}\left(1-|\varphi|^{2}\right)-A_{n} .
\end{aligned}
$$

Then, invoking the dominated convergence theorem,

$$
A_{n}=\frac{1}{4} \int_{\mathbb{R}^{N}} \chi_{n}\left(1-|\varphi|^{2}\right)^{2}+\frac{1}{4} \int_{\mathbb{R}^{N}} x_{j} \partial_{j} \chi_{n}\left(1-|\varphi|^{2}\right) \partial_{j}\left(1-|\varphi|^{2}\right) \rightarrow \frac{1}{4} \int_{\mathbb{R}^{N}}\left(1-|\varphi|^{2}\right)^{2},
$$

as $n \rightarrow \infty$. In particular, we see that due to a symmetry property, we can write $A_{n}$ in terms of integrals to which we can apply the dominated convergence theorem. However, in our nonlocal case we cannot use this trick and we have to analyze the integral associated to the potential energy more carefully. We rely in particular on the following general result.

Proposition 5.3. Let $f \in L^{2}\left(\mathbb{R}^{N}\right) \cap H_{\mathrm{loc}}^{1}\left(\mathbb{R}^{N}\right)$ be a real-valued function and $W \in$ $\mathcal{M}_{2,2}\left(\mathbb{R}^{N}\right)$. Assume also that $(\mathrm{H} 1)$ and $(\mathrm{H} 3)$ hold. Then, for all $j \in\{1, \ldots, N\}$,

$\lim _{n \rightarrow \infty}-\frac{1}{2} \int_{\mathbb{R}^{N}}(W * f) x_{j} \chi_{n} \partial_{j} f=\frac{1}{4} \int_{\mathbb{R}^{N}}(W * f) f-\frac{1}{4(2 \pi)^{N}} \int_{\mathbb{R}^{N}} \xi_{j} \partial_{j} \widehat{W}(\xi)|\widehat{f}(\xi)|^{2} d \xi$.

The proof of Proposition 5.3 is rather technical, so that we postpone it. Assuming the result, we now give the proof of the Pohozaev identity.

Proof of Proposition 5.1 assuming Proposition 5.3. By putting together (5.3)-(5.5) (with $\varphi=v$ ) and Proposition 5.3 , we have for $j \in\{1, \ldots, N\}$,

$$
\begin{aligned}
\frac{1}{2} \int_{\mathbb{R}^{N}}|\nabla v|^{2}+\frac{1}{4} \int_{\mathbb{R}^{N}}(W * \eta) \eta= & \int_{\mathbb{R}^{N}}\left|\partial_{j} v\right|^{2}-\left(1-\delta_{1, j}\right) \frac{c}{2} \int_{\mathbb{R}^{N}}\left(v_{1} \partial_{1} v_{2}-v_{2} \partial_{1} v_{1}-\partial_{1}(\chi \theta)\right) \\
& +\frac{1}{4(2 \pi)^{N}} \int_{\mathbb{R}^{N}} \xi_{j} \partial_{j} \widehat{W}(\xi)|\widehat{\eta}(\xi)|^{2} d \xi
\end{aligned}
$$

which is exactly (5.1)-(5.2).

We remark that the main problem in order to establish the convergence in (5.7) is that $f$ does not decay fast enough at infinity. Indeed, let us suppose that $x_{j} f, x_{j} \partial_{j} f \in L^{2}\left(\mathbb{R}^{N}\right)$. Then by the dominated convergence theorem and the Plancherel identity we have

$$
B_{n}:=-\frac{1}{2} \int_{\mathbb{R}^{N}}(W * f) x_{j} \chi_{n} \partial_{j} f \rightarrow-\frac{1}{2(2 \pi)^{N}} \int_{\mathbb{R}^{N}} \widehat{W} \overline{\widehat{f}} \widehat{x_{j} \partial_{j} f}, \quad \text { as } n \rightarrow \infty .
$$

Using (1.22), we conclude that

$$
\begin{aligned}
\lim _{n \rightarrow \infty} B_{n} & =\frac{1}{2(2 \pi)^{N}} \int_{\mathbb{R}^{N}} \widehat{W}|\widehat{f}|^{2}+\frac{1}{2(2 \pi)^{N}} \int_{\mathbb{R}^{N}} \widehat{W} \xi_{j} \bar{f} \partial_{j} \widehat{f} \\
& =\frac{1}{4} \int_{\mathbb{R}^{N}}(W * f) f-\frac{1}{4(2 \pi)^{N}} \int_{\mathbb{R}^{N}} \xi_{j} \partial_{j} \widehat{W}|\widehat{f}|^{2},
\end{aligned}
$$


where we have used the Plancherel identity, integration by parts and that $\partial_{j} \widehat{f} \in$ $L^{2}\left(\mathbb{R}^{N}\right)$. This yields (5.7), but only under these more restrictive assumptions. If we only have that $f \in L^{2}\left(\mathbb{R}^{N}\right) \cap H_{\text {loc }}^{1}\left(\mathbb{R}^{N}\right)$, we can neither invoke the dominated convergence theorem nor justify that the second integral in the r.h.s. of (5.8) is finite. Therefore, to deal with the limit $n \rightarrow \infty$ in Proposition 5.3, we first establish the following lemma.

Lemma 5.4. Let $g \in L^{2}\left(\mathbb{R}^{N}\right)$ and $F \in L^{\infty}\left(\mathbb{R}^{N} \times \mathbb{R}^{N}\right)$. Assume also that $F(\cdot, 0) \in$ $L^{\infty}\left(\mathbb{R}^{N}\right)$ and that

$$
F\left(\xi, r_{n}\right) \rightarrow F(\xi, 0), \quad \text { as }\left|r_{n}\right| \rightarrow 0, \text { for a.a. } \xi \in \mathbb{R}^{N} .
$$

For $\varphi \in C_{0}^{\infty}\left(\mathbb{R}^{N}\right)$, we set

$$
\widehat{\varphi}_{n}(\xi):=n^{N} \widehat{\varphi}(n \xi) \quad \text { and } \Psi_{n}(\xi):=\int_{\mathbb{R}^{N}} F(\xi, r) g(\xi-r) \widehat{\varphi}_{n}(r) d r
$$

for a.a. $\xi \in \mathbb{R}^{N}$. Then

$$
\Psi_{n} \rightarrow(2 \pi)^{N} F(\cdot, 0) g(\cdot) \varphi(0), \quad \text { in } L^{2}\left(\mathbb{R}^{N}\right) \text {, as } n \rightarrow \infty .
$$

Proof. Let

$$
\Psi(\xi):=(2 \pi)^{N} F(\xi, 0) g(\xi) \varphi(0), \quad \text { for a.a. } \xi \in \mathbb{R}^{N} .
$$

We notice that by (5.10)

$$
\int_{\mathbb{R}^{N}} \widehat{\varphi}_{n}(r) d r=\int_{\mathbb{R}^{N}} \widehat{\varphi}(r) d r=(2 \pi)^{N} \varphi(0),
$$

so that

$$
\Psi_{n}(\xi)-\Psi(\xi)=\int_{\mathbb{R}^{N}}(F(\xi, r) g(\xi-r)-F(\xi, 0) g(\xi)) \widehat{\varphi}_{n}(r) d r
$$

Then

$$
\begin{aligned}
\left|\Psi_{n}(\xi)-\Psi(\xi)\right| \leq & \|F\|_{L^{\infty}\left(\mathbb{R}^{2 N}\right)} \int_{\mathbb{R}^{N}}|g(\xi-r)-g(\xi)|\left|\widehat{\varphi}_{n}(r)\right| d r \\
& +|g(\xi)| \int_{\mathbb{R}^{N}}\left|F(\xi, r)-F(\xi, 0) \| \widehat{\varphi}_{n}(r)\right| d r .
\end{aligned}
$$

On the other hand, using (5.10) and integrating by parts, we are led to

$$
\begin{aligned}
\left|\widehat{\varphi}_{n}(\xi)\right| & =n^{N}\left|\int_{\mathbb{R}^{N}} \varphi(y) e^{-i n \xi \cdot y} d y\right| \\
& =\frac{n^{N-2 l}}{|\xi|^{2 l}}\left|\int_{\mathbb{R}^{N}} \Delta^{l} \varphi(y) e^{-i n \xi \cdot y} d y\right| \leq \frac{n^{N-2 l}}{|\xi|^{2 l}}\left\|\Delta^{l} \varphi\right\|_{L^{1}\left(\mathbb{R}^{N}\right)},
\end{aligned}
$$

for any $l \in \mathbb{N}$ and any $\xi \neq 0$. Invoking this estimate for $l=N$ and the Minkowski integral inequality, we get

$$
\begin{aligned}
\left\|\int _ { B ( 0 , 1 / \sqrt { n } ) ^ { c } } \left|g(\xi-r)-g(\xi)\left\|\widehat{\varphi}_{n}(r) \mid d r\right\|_{L^{2}\left(\mathbb{R}^{N}\right)}\right.\right. & \leq 2\|g\|_{L^{2}\left(\mathbb{R}^{N}\right)}\left\|\widehat{\varphi}_{n}\right\|_{L^{1}\left(B(0,1 / \sqrt{n})^{c}\right)} \\
& \leq \frac{C(N, \varphi)}{n^{N / 2}}\|g\|_{L^{2}\left(\mathbb{R}^{N}\right) .}
\end{aligned}
$$


Similarly, we obtain

$\left\||g(\xi)| \int_{B(0,1 / \sqrt{n})^{c}}\left|F(\xi, r)-F(\xi, 0)\left\|\widehat{\varphi}_{n}(r) \mid d r\right\|_{L^{2}\left(\mathbb{R}^{N}\right)} \leq \frac{C(N, \varphi)}{n^{N / 2}}\|F\|_{L^{\infty}\left(\mathbb{R}^{2 N}\right)}\|g\|_{L^{2}\left(\mathbb{R}^{N}\right)}\right.\right.$.

On the other hand, using again the Minkowski integral inequality and (5.10),

$$
\begin{aligned}
\| \int_{B(0,1 / \sqrt{n})} \mid g(\xi-r)-g(\xi) & \left\|\widehat{\varphi}_{n}(r) \mid d r\right\|_{L^{2}\left(\mathbb{R}^{N}\right)} \\
& \leq\|\| g(\cdot-r)-g\left\|_{L^{2}\left(\mathbb{R}^{N}\right)}\left|\widehat{\varphi}_{n}(r)\right|\right\|_{L^{1}(B(0,1 / \sqrt{n}))} \\
& \leq \sup _{|y| \leq 1 / \sqrt{n}}\|g(\cdot-y)-g\|_{L^{2}\left(\mathbb{R}^{N}\right)}\left\|\widehat{\varphi}_{n}\right\|_{L^{1}(B(0,1 / \sqrt{n}))} \\
& \leq \sup _{|y| \leq 1 / \sqrt{n}}\|g(\cdot-y)-g\|_{L^{2}\left(\mathbb{R}^{N}\right)} \| \widehat{\varphi}_{L^{1}\left(\mathbb{R}^{N}\right)} .
\end{aligned}
$$

Since $g \in L^{2}\left(\mathbb{R}^{N}\right)$, we know that

$$
\sup _{|y| \leq h}\|g(\cdot-y)-g\|_{L^{2}\left(\mathbb{R}^{N}\right)} \rightarrow 0, \text { as } h \rightarrow 0,
$$

so that

$$
\left\|\int _ { B ( 0 , 1 / \sqrt { n } ) } \left|g(\xi-r)-g(\xi)\left\|\widehat{\varphi}_{n}(r) \mid d r\right\|_{L^{2}\left(\mathbb{R}^{N}\right)} \rightarrow 0, \text { as } n \rightarrow+\infty .\right.\right.
$$

We now turn to the second term in the r.h.s. of (5.13). By a change of variables, we get that it is equal to

$$
|g(\xi)| \int_{B(0, \sqrt{n})}|F(\xi, r / n)-F(\xi, 0)||\widehat{\varphi}(r)| d r .
$$

Since $\widehat{\varphi} \in L^{1}\left(\mathbb{R}^{N}\right)$ and

$$
|F(\xi, r / n)-F(\xi)||\widehat{\varphi}(r)| \leq 2\|F\|_{L^{\infty}\left(\mathbb{R}^{2 N}\right)}|\widehat{\varphi}(r)|,
$$

we can deduce from (5.9) and the dominated convergence theorem that

$$
\int_{B(0, \sqrt{n})}|F(\xi, r / n)-F(\xi) \| \widehat{\varphi}(r)| d r \rightarrow 0, \text { as } n \rightarrow+\infty,
$$

for a.a. $\xi \in \mathbb{R}^{N}$. On the other hand,

$$
|g(\xi)| \int_{B(0, \sqrt{n})}\left|F(\xi, r / n)-F(\xi)\left\|\widehat{\varphi}(r)\left|d r \leq 2\|F\|_{L^{\infty}\left(\mathbb{R}^{2 N}\right)}\|\widehat{\varphi}\|_{L^{1}\left(\mathbb{R}^{N}\right)}\right| g(\xi) \mid,\right.\right.
$$

Therefore, again by the dominated convergence theorem,

$$
\left\||g(\xi)| \int_{B(0, \sqrt{n})}|F(\xi, r / n)-F(\xi)||\widehat{\varphi}(r)| d r\right\|_{L^{2}\left(\mathbb{R}^{N}\right)} \rightarrow 0 \text {, as } n \rightarrow+\infty .
$$

By combining with (5.13) (5.17), we conclude (5.11), which finishes the proof of Lemma 5.4

Proof of Proposition 5.3. Setting $W_{m}=\mathcal{F}^{-1}\left(\chi_{m} \widehat{W}\right)=\mathcal{F}^{-1}\left(\chi_{m}\right) * W$, we have that $W_{m}$ is even, $W_{m} \in C^{\infty}\left(\mathbb{R}^{N}\right)$,

$$
\widehat{W}_{m} \rightarrow \widehat{W}, \nabla \widehat{W}_{m} \rightarrow \nabla \widehat{W} \text { a.e. and } W_{m} * g \rightarrow W * g \text { in } L^{2}\left(\mathbb{R}^{N}\right),
$$


for all $g \in L^{2}\left(\mathbb{R}^{N}\right)$, as $m \rightarrow \infty$. Therefore

$$
I_{n, m}:=-\frac{1}{2} \int_{\mathbb{R}^{N}}\left(W_{m} * f\right) x_{j} \chi_{n} \partial_{j} f \underset{m \rightarrow \infty}{\longrightarrow} I_{n}:=-\frac{1}{2} \int_{\mathbb{R}^{N}} \chi_{n}(W * f) x_{j} \partial_{j} f .
$$

Moreover, since the Fourier transform of all derivatives of $W_{m}$ have compact support, they are bounded in $L^{2}\left(\mathbb{R}^{N}\right)$. Then, by the Plancherel theorem, we conclude that

$$
W_{m} \in W^{k, 2}\left(\mathbb{R}^{N}\right), \quad \text { for all } k \in \mathbb{N} .
$$

In particular, this implies that $W_{m} * f$ belongs to $C^{1}\left(\mathbb{R}^{N}\right) \cap L^{2}\left(\mathbb{R}^{N}\right)$, with

$$
\partial_{j}\left(W_{m} * f\right)=\partial_{j} W_{m} * f
$$

Thus, integrating by parts, we have that

$$
I_{n, m}=P_{n, m}+Q_{n, m}
$$

where

$$
P_{n, m}=\frac{1}{2} \int_{\mathbb{R}^{N}}\left(\partial_{j} W_{m} * f\right) x_{j} \chi_{n} f \text { and } Q_{n, m}=\frac{1}{2} \int_{\mathbb{R}^{N}}\left(W_{m} * f\right)\left(\chi_{n}+x_{j} \partial_{j} \chi_{n}\right) f .
$$

By (5.18),

$$
\lim _{m \rightarrow \infty} Q_{n, m}=\frac{1}{2} \int_{\mathbb{R}^{N}}(W * f)\left(\chi_{n}+x_{j} \partial_{j} \chi_{n}\right) f .
$$

Since $\left|x_{j} \partial_{j} \chi(x)\right| \leq 2\left\|\chi^{\prime}\right\|_{L^{\infty}(\mathbb{R})}$, by the dominated convergence theorem,

$$
\lim _{n \rightarrow \infty} \int_{\mathbb{R}^{N}}(W * f)\left(\chi_{n}+x_{j} \partial_{j} \chi_{n}\right) f=\int_{\mathbb{R}^{N}}(W * f) f
$$

On the other hand, by the Cauchy-Schwarz inequality,

$$
\int_{\mathbb{R}^{N}}\left|\partial_{j} W_{m}(x-y) f(y)\right| d y \leq\left\|\partial_{j} W_{m}\right\|_{L^{2}\left(\mathbb{R}^{N}\right)}\|f\|_{L^{2}\left(\mathbb{R}^{N}\right)}, \quad x \in \mathbb{R}^{N},
$$

so that

$\int_{\mathbb{R}^{N}} \int_{\mathbb{R}^{N}}\left|\partial_{j} W_{m}(x-y) f(y) x_{j} f(x) \chi_{n}(x)\right| d y d x \leq 2 n\left\|\partial_{j} W_{m}\right\|_{L^{2}\left(\mathbb{R}^{N}\right)}\|f\|_{L^{2}\left(\mathbb{R}^{N}\right)}^{2}\left\|\chi_{n}\right\|_{L^{2}\left(\mathbb{R}^{N}\right)}$.

Since $W_{m}$ is an even function, $\partial W_{m}$ is odd. Then, by (5.23) we can use the Fubini theorem to deduce that

$$
P_{n, m}=\frac{1}{4} \int_{\mathbb{R}^{N}} \int_{\mathbb{R}^{N}} \partial_{j} W_{m}(x-y) f(y) f(x)\left(x_{j} \chi_{n}(x)-y_{j} \chi_{n}(y)\right) d y d x
$$

Let us denote

$$
G_{n, m}(x):=\int_{\mathbb{R}^{N}} \partial_{j} W_{m}(x-y) f(y)\left(x_{j} \chi_{n}(x)-y_{j} \chi_{n}(y)\right) d y,
$$

for a.a. $x \in \mathbb{R}^{N}$. Arguing as before, using the Young inequality and (15.20), we have $\left\|G_{n, m}\right\|_{L^{1}\left(\mathbb{R}^{N}\right)} \leq\left\|\partial_{j} W_{m}\right\|_{L^{2}\left(\mathbb{R}^{N}\right)}\|f\|_{L^{2}\left(\mathbb{R}^{N}\right)}\left\|x_{j} \chi_{n}\right\|_{L^{1}\left(\mathbb{R}^{N}\right)}+\left\|\partial_{j} W_{m}\right\|_{L^{\infty}\left(\mathbb{R}^{N}\right)}\left\|f x_{j} \chi_{n}\right\|_{L^{1}\left(\mathbb{R}^{N}\right)}$, $\left\|G_{n, m}\right\|_{L^{2}\left(\mathbb{R}^{N}\right)} \leq\left\|\partial_{j} W_{m}\right\|_{L^{2}\left(\mathbb{R}^{N}\right)}\|f\|_{L^{2}\left(\mathbb{R}^{N}\right)}\left\|x_{j} \chi_{n}\right\|_{L^{2}\left(\mathbb{R}^{N}\right)}+\left\|\partial_{j} W_{m}\right\|_{L^{2}\left(\mathbb{R}^{N}\right)}\left\|f x_{j} \chi_{n}\right\|_{L^{1}\left(\mathbb{R}^{N}\right)}$.

Thus $G_{n, m} \in L^{1}\left(\mathbb{R}^{N}\right) \cap L^{2}\left(\mathbb{R}^{N}\right)$. Moreover, since the function $x \mapsto x_{j} \chi_{n}(x)$ is smooth on $\mathbb{R}^{N}$, we can write

$$
x_{j} \chi_{n}(x)-y_{j} \chi_{n}(y)=\sum_{k=1}^{N}\left(x_{k}-y_{k}\right) \theta_{k}(y, x-y),
$$


where

$$
\theta_{k}(y, z):=\int_{0}^{1}\left(\delta_{j, k} \chi_{n}(y+t z)+\left(y_{j}+t z_{j}\right) \partial_{k} \chi_{n}(y+t z)\right) d t .
$$

Therefore, the function $G_{n, m}$ may be written almost everywhere as

$$
G_{n, m}(x)=\sum_{k=1}^{N} \int_{\mathbb{R}^{N}}\left(x_{k}-y_{k}\right) \partial_{j} W_{m}(x-y) f(y) \theta_{k}(y, x-y) d y,
$$

so that its Fourier transform is equal to

$$
\begin{aligned}
\widehat{G}_{n, m}(p) & =\sum_{k=1}^{N} \int_{\mathbb{R}^{N}} \int_{\mathbb{R}^{N}}\left(x_{k}-y_{k}\right) \partial_{j} W_{m}(x-y) f(y) \theta_{k}(y, x-y) e^{-i p \cdot x} d y d x \\
& =\sum_{k=1}^{N} \int_{\mathbb{R}^{N}} \int_{\mathbb{R}^{N}} z_{k} \partial_{j} W_{m}(z) f(y) \theta_{k}(y, z) e^{-i p \cdot(y+z)} d y d z \\
& =\frac{1}{(2 \pi)^{N}} \sum_{k=1}^{N} \int_{\mathbb{R}^{N}} \int_{\mathbb{R}^{N}} z_{k} \partial_{j} W_{m}(z) \widehat{f}(p-r) \tilde{\theta}_{k}(r, z) e^{-i p \cdot z} d r d z
\end{aligned}
$$

where

$$
\begin{aligned}
\tilde{\theta}_{k}(r, z) & :=\int_{\mathbb{R}^{N}} \theta_{k}(y, z) e^{-i r \cdot y} d y \\
& =\int_{\mathbb{R}^{N}}\left(\int_{0}^{1}\left(\delta_{j, k} \chi_{n}(y+t z)+\left(y_{j}+t z_{j}\right) \partial_{k} \chi_{n}(y+t z)\right) d t\right) \theta_{k}(y, z) e^{-i r \cdot y} d y \\
& =\int_{0}^{1} e^{i t r . z}\left(\delta_{j, k} \widehat{\chi}_{n}(r)+\widehat{y_{j} \partial_{k} \chi_{n}}(r)\right) d t .
\end{aligned}
$$

Hence, we are led to

$$
\widehat{G}_{n, m}(p)=\frac{1}{(2 \pi)^{N}} \sum_{k=1}^{N} \int_{\mathbb{R}^{N}} \int_{0}^{1} z_{k} \widehat{\partial}_{j} W_{m}(p-r t) \widehat{f}(p-r)\left(\delta_{j, k} \widehat{\chi_{n}}(r)+\widehat{y_{j} \partial_{k} \chi_{n}}(r)\right) d t d r .
$$

At this stage, we note that by (5.18) and (1.22),

$$
z_{k} \widehat{\partial j}_{m}(p) \rightarrow \widehat{z_{k} \partial_{j} W}(p)=-p_{j} \partial_{k} \widehat{W}(p)-\delta_{k, j} \widehat{W}(p) \text { a.e, as } m \rightarrow+\infty,
$$

whereas

$$
\left|z_{k} \widehat{\partial}_{j} W_{m}(p)\right| \leq\left(1+2\left\|\chi^{\prime}\right\|_{L^{\infty}(\mathbb{R})}\right)\|\widehat{W}\|_{L^{\infty}\left(\mathbb{R}^{N}\right)}+\left\|p_{j} \partial_{k} \widehat{W}\right\|_{L^{\infty}\left(\mathbb{R}^{N}\right)}
$$

for a.a. $p \in \mathbb{R}^{N}$. Invoking the dominated convergence theorem, we deduce that

$$
\widehat{G}_{n, m}(p) \rightarrow \widehat{G}_{n}(p), \quad \text { as } m \rightarrow+\infty,
$$

for a.a. $p \in \mathbb{R}^{N}$, where

$$
\widehat{G}_{n}(p):=\frac{1}{(2 \pi)^{N}} \sum_{k=1}^{N} \int_{\mathbb{R}^{N}} \int_{0}^{1} \widehat{z_{k} \partial_{j} W}(p-r t) \widehat{f}(p-r)\left(\delta_{j, k} \widehat{\chi}_{n}(r)+\widehat{y_{j} \partial_{k} \chi_{n}}(r)\right) d t d r .
$$


Moreover, since

$$
\begin{array}{r}
\left|\widehat{G_{n, m}}(p)\right| \leq \frac{1}{(2 \pi)^{N}} \sum_{k=1}^{N}\left(\left(1+2\left\|\chi^{\prime}\right\|_{L^{\infty}(\mathbb{R})}\right)\|\widehat{W}\|_{L^{\infty}\left(\mathbb{R}^{N}\right)}+\left\|p_{j} \partial_{j} \widehat{W}\right\|_{L^{\infty}\left(\mathbb{R}^{N}\right)}\right) \times \\
\quad \times \int_{\mathbb{R}^{N}}|\widehat{f}(p-r)|\left|\delta_{j, k} \widehat{\chi_{n}}(r)+\widehat{y_{j} \partial_{k} \chi_{n}}(r)\right| d r,
\end{array}
$$

it follows again from the dominated convergence theorem that

$$
\widehat{G}_{n, m} \rightarrow \widehat{G}_{n} \text { in } L^{2}\left(\mathbb{R}^{N}\right) \text {, as } m \rightarrow+\infty .
$$

Hence, recalling (5.24) and (5.25), we are led to

$$
P_{n, m} \rightarrow P_{n}:=\frac{1}{4} \int_{\mathbb{R}^{N}} G_{n}(x) f(x) d x, \text { as } m \rightarrow+\infty .
$$

Finally, since

$$
\begin{gathered}
\widehat{\chi}_{n}(p)=n^{N} \int_{\mathbb{R}^{N}} \chi_{1}(y) e^{-i n p \cdot y} d y=n^{N} \widehat{\chi}_{1}(p), \\
\widehat{y_{j} \partial_{k} \chi_{n}}(p)=n^{N} \int_{\mathbb{R}^{N}} y_{j} \partial_{k} \chi_{1}(y) e^{-i n p \cdot y} d y=n^{N} \widehat{y_{j} \partial_{k} \chi_{1}}(n p),
\end{gathered}
$$

$\chi_{1}=1$ and $\partial_{k} \chi_{1}=0$ on $B(0,1)$, applying Lemma 5.4 with

$$
\varphi=\delta_{j, k} \chi_{1}+y_{j} \partial_{k} \chi_{1}, \quad F(p, r)=\int_{0}^{1} \widehat{z_{k} \partial_{j} W}(p-r t) d t, \quad g=\widehat{f},
$$

we conclude that

$$
\widehat{G}_{n} \rightarrow \widehat{z_{j} \partial_{j} W} \widehat{f} \text { in } L^{2}\left(\mathbb{R}^{N}\right), \quad \text { as } n \rightarrow \infty .
$$

Therefore, in view of (5.26), (5.27) and the Plancherel identity, we have

$$
P_{n} \rightarrow \frac{1}{4(2 \pi)^{N}} \int_{\mathbb{R}^{N}} \widehat{z_{j} \partial_{j} W}(p)|\widehat{f}(p)|^{2} d p \text {, as } n \rightarrow+\infty .
$$

By combining with (1.22), (5.19), (5.21), (5.22) and (5.26), we obtain (5.7).

\section{Proof of the Main Results}

We are now in position to provide the proofs of the results stated in Subsection 1.4 .

Proof of Theorem 1.8, For $j \in\{1, \ldots, N\}$, let us introduce the notation

$$
\begin{aligned}
\mathcal{K}_{j} & :=\frac{1}{2} \int_{\mathbb{R}^{N}}\left|\partial_{j} v\right|^{2}, \quad \mathcal{K}:=\sum_{j=1}^{N} \mathcal{K}_{j}, \quad \mathcal{R}_{j}:=\frac{1}{4(2 \pi)^{N}} \int_{\mathbb{R}^{N}} \xi_{j} \partial_{j} \widehat{W}|\widehat{\eta}|^{2}, \\
\mathcal{P} & :=\int_{\mathbb{R}^{N}}\left(v_{1} \partial_{1} v_{2}-v_{2} \partial_{1} v_{1}-\partial_{1}(\chi \theta)\right), \quad \mathcal{U}:=\frac{1}{4} \int_{\mathbb{R}^{N}}(W * \eta) \eta .
\end{aligned}
$$

In this way

$$
E(v)=\mathcal{K}+\mathcal{U}
$$


and Propositions 3.1 and $5.1 \mathrm{read}$

$$
\begin{aligned}
\mathcal{K}+2 \mathcal{U} & =-\frac{c \ell_{c}}{2\left(1+\ell_{c}\right)} \mathcal{P}, \\
\mathcal{K}+\mathcal{U} & =2 \mathcal{K}_{1}+\mathcal{R}_{1}, \\
\mathcal{K}+\mathcal{U} & =2 \mathcal{K}_{j}-\frac{c}{2} \mathcal{P}+\mathcal{R}_{j},
\end{aligned}
$$

for all $j \in\{2, \ldots, N\}$. From (6.2) and (6.4), we obtain

$$
\left(1+2 \ell_{c}\right) \mathcal{K}_{j}+\sum_{\substack{k=1 \\ k \neq j}}^{N} \mathcal{K}_{k}+\left(\ell_{c}+2\right) \mathcal{U}=-\ell_{c} \mathcal{R}_{j}, \quad j \in\{2, \ldots, N\} .
$$

Therefore, we can write (6.1), (6.3) and (6.5) as the linear system $A z=b$, with

$$
z=\left(\mathcal{K}_{1}, \mathcal{K}_{2}, \ldots, \mathcal{K}_{n}, \mathcal{U}\right), \quad b=\left(\mathcal{R}_{1},-\ell_{c} \mathcal{R}_{2}, \ldots,-\ell_{c} \mathcal{R}_{N}, E(v)\right)
$$

and $A \in \mathbb{R}^{N+1 \times N+1}$ given by

$$
A_{i, j}= \begin{cases}-1, & \text { if } i=j=1, \\ 2+\ell_{c}, & \text { if } j=N+1,1<i<N+1, \\ 1+2 \ell_{c}, & \text { if } i=j, i \neq 1, \\ 1, & \text { otherwise. }\end{cases}
$$

Let $\sigma=\left(\sigma_{1}, \sigma_{2}, \ldots, \sigma_{N},-1\right)$. If $K(v)=0, v$ is constant. Therefore we suppose that $K(v)>0$. Then using (1.16),

$$
\begin{aligned}
b^{T} \sigma & =\sigma_{1} \mathcal{R}_{1}-\ell_{c} \sum_{k=2}^{N} \sigma_{k} \mathcal{R}_{k}-E(v) \\
& =\frac{1}{4(2 \pi)^{N}} \int_{\mathbb{R}^{N}}|\widehat{\eta}(\xi)|^{2}\left(\sigma_{1} \xi_{1} \partial_{1} \widehat{W}(\xi)-\ell_{c} \sum_{k=2}^{N} \sigma_{k} \xi_{k} \partial_{k} \widehat{W}(\xi)-\widehat{W}(\xi)\right) d \xi-K(v) \\
& \leq-K(v)<0 .
\end{aligned}
$$

On the other hand,

$$
\left(A^{T} \sigma\right)_{j}= \begin{cases}-\sigma_{1}+\sum_{k=2}^{N} \sigma_{k}-1, & \text { if } j=1, \\ \sigma_{1}+\sum_{k=2}^{N} \sigma_{k}+2 \ell_{c} \sigma_{j}-1, & \text { if } 2 \leq j \leq N, \\ \sigma_{1}+\left(\ell_{c}+2\right) \sum_{k=2}^{N} \sigma_{k}-1, & \text { if } 2 \leq j=N+1 .\end{cases}
$$

Consequently, by (1.17), $A^{T} \sigma \geq 0$. However, since $z \geq 0$, this inequality together with (6.6) contradict Farkas' Lemma.

Proof of Theorem 1.3. It is an immediate consequence of Theorem 1.8 and Lemma 4.1 .

Proof of Theorem 1.6. Using the notation of the proof of Theorem 1.8, by (1.12) and Proposition 5.1 we conclude that

$$
\mathcal{K}+\mathcal{U} \leq 2 \mathcal{K}_{j}, \quad \text { for all } j \in\{1, \ldots, N\} .
$$


Thus, summing over $j$,

$$
\mathcal{U} \leq \frac{2-N}{N} \mathcal{K}
$$

Since $N \geq 2, \mathcal{K} \geq 0$ and $\mathcal{U} \geq 0$, inequality (6.7) implies that $\mathcal{U}=0$ and therefore $v$ is constant.

Proof of Corollary 1.4. Let us take $\sigma_{1}=-1$ and $\bar{\sigma}:=\sigma_{2}=\cdots=\sigma_{N}>0$. In order to fulfill (1.9), we finally fix

$$
\bar{\sigma}=\max \left\{\frac{2}{(N-1)\left(\alpha_{c}+2\right)}, \frac{2}{N-1+\alpha_{c}}\right\} .
$$

Then $\alpha_{c} \bar{\sigma} \leq \max \{1,2 /(N-1)\}$, so that

$$
\widehat{W}(\xi)+\alpha_{c} \sum_{k=2}^{N} \sigma_{k}\left|\xi_{k} \partial_{k} \widehat{W}(\xi)\right| \geq \widehat{W}(\xi)-\max \left\{1, \frac{2}{N-1}\right\} \sum_{k=2}^{N}\left|\xi_{k} \partial_{k} \widehat{W}(\xi)\right|-\left|\xi_{1} \partial_{1} \widehat{W}(\xi)\right| .
$$

Therefore the conclusion follows from (1.10) and Theorem 1.3 .

Proof of Corollary 1.5. Taking $\sigma_{1}=0$ and $\sigma_{2}=\cdots=\sigma_{N}=1 /(N-1)$, we have that (1.9) is satisfied. Let

$$
m:=\inf _{\xi \in \mathbb{R}^{N}} \frac{(N-1) \widehat{W}(\xi)}{\sum_{k=2}^{N}\left|\xi_{k} \partial_{k} \widehat{W}(\xi)\right|} .
$$

If $m=+\infty, \xi_{j} \partial_{j} \widehat{W}(\xi)=0$ a.e. for all $j \in\{2, \ldots, N\}$ and then (1.8) is fulfilled. If $m<\infty$, we note that (1.11) implies $\alpha_{c} \leq m$, so that

$$
\widehat{W}(\xi)+\alpha_{c} \sum_{k=2}^{N} \sigma_{k}\left|\xi_{k} \partial_{k} \widehat{W}(\xi)\right| \geq \frac{m-\alpha_{c}}{N-1} \sum_{k=2}^{N}\left|\xi_{k} \partial_{k} \widehat{W}(\xi)\right| \geq 0 .
$$

Then Theorem 1.3 yields the conclusion.

Proof of Corollary 1.9. The proof is analogous to that of Corollaries 1.4 and 1.5. The only difference is that we invoke Theorem 1.8 instead of Theorem 1.3 to conclude.

\section{REFERENCES}

[1] A. Aftalion, X. Blanc, and R. Jerrard. Nonclassical rotational inertia of a supersolid. Phys. Rev. Lett., 99(13):135301.1-135301.4, 2007.

[2] N. G. Berloff and P. H. Roberts. Motions in a Bose condensate VI. Vortices in a nonlocal model. J. Phys. A, 32(30):5611-5625, 1999.

[3] F. Béthuel, P. Gravejat, and J.-C. Saut. Existence and properties of travelling waves for the Gross-Pitaevskii equation. In A. Farina and J.-C. Saut, editors, Stationary and time dependent Gross-Pitaevskii equations. Wolfgang Pauli Institute 2006 thematic program, JanuaryDecember, 2006, Vienna, Austria, volume 473 of Contemporary Mathematics, pages 55-104. American Mathematical Society.

[4] F. Béthuel, P. Gravejat, and J.-C. Saut. Travelling waves for the Gross-Pitaevskii equation. II. Comm. Math. Phys., 285(2):567-651, 2009.

[5] F. Bethuel, G. Orlandi, and D. Smets. Vortex rings for the Gross-Pitaevskii equation. J. Eur. Math. Soc. (JEMS), 6(1):17-94, 2004.

[6] F. Béthuel and J.-C. Saut. Travelling waves for the Gross-Pitaevskii equation I. Ann. Inst. H. Poincaré Phys. Théor., 70(2):147-238, 1999. 
[7] N. N. Bogoliubov. On the theory of superfluidity. J. Phys. USSR, 11:23-32, 1947. Reprinted in: D. Pines, The Many-Body Problem (W. A. Benjamin, New York, 1961), p. 292-301.

[8] H. Brezis, F. Merle, and T. Rivière. Quantization effects for $-\Delta u=u\left(1-|u|^{2}\right)$ in $\mathbf{R}^{2}$. Arch. Rational Mech. Anal., 126(1):35-58, 1994.

[9] R. Carles, P. A. Markowich, and C. Sparber. On the Gross-Pitaevskii equation for trapped dipolar quantum gases. Nonlinearity, 21(11):2569-2590, 2008.

[10] D. Chiron. Travelling waves for the Gross-Pitaevskii equation in dimension larger than two. Nonlinear Anal., 58(1-2):175-204, 2004.

[11] C. Coste. Nonlinear Schrödinger equation and superfluid hydrodynamics. Eur. Phys. J. B Condens. Matter Phys., 1(2):245-253, 1998.

[12] J. Cuevas, B. A. Malomed, P. G. Kevrekidis, and D. J. Frantzeskakis. Solitons in quasi-onedimensional Bose-Einstein condensates with competing dipolar and local interactions. Phys. Rev. A, 79(5):053608.1-053608.11, 2009.

[13] A. de Laire. Global well-posedness for a nonlocal Gross-Pitaevskii equation with non-zero condition at infinity. Comm. Partial Differential Equations, 35(11):2021-2058, 2010.

[14] B. Deconinck and J. N. Kutz. Singular instability of exact stationary solutions of the non-local Gross-Pitaevskii equation. Phys. Lett. A, 319(1-2):97-103, 2003.

[15] L. Grafakos. Classical Fourier analysis, volume 249 of Graduate Texts in Mathematics. Springer, New York, second edition, 2008.

[16] P. Gravejat. A non-existence result for supersonic travelling waves in the Gross-Pitaevskii equation. Comm. Math. Phys., 243(1):93-103, 2003.

[17] P. Gravejat. Limit at infinity and nonexistence results for sonic travelling waves in the GrossPitaevskii equation. Differential Integral Equations, 17(11-12):1213-1232, 2004.

[18] E. Gross. Hydrodynamics of a superfluid condensate. J. Math. Phys., 4(2):195-207, 1963.

[19] L. Hörmander. The analysis of linear partial differential operators I. Classics in Mathematics. Springer-Verlag, Berlin, 2003.

[20] C. A. Jones, S. J. Putterman, and P. H. Roberts. Motions in a Bose condensate V. Stability of solitary wave solutions of non-linear Schrödinger equations in two and three dimensions. J. Phys. A, Math. Gen., 19(15):2991-3011, 1986.

[21] C. A. Jones and P. H. Roberts. Motions in a Bose condensate IV. Axisymmetric solitary waves. J. Phys. A, Math. Gen., 15(8):2599-2619, 1982.

[22] C. Josserand, Y. Pomeau, and S. Rica. Coexistence of ordinary elasticity and superfluidity in a model of a defect-free supersolid. Phys. Rev. Lett., 98(19):195301.1-195301.4, 2007.

[23] Y. S. Kivshar and B. Luther-Davies. Dark optical solitons: physics and applications. Phys. Rep., 298(2-3):81-197, 1998.

[24] M. Mariş. Traveling waves for nonlinear Schrödinger equations with nonzero conditions at infinity. Preprint arXiv 0903.0354.

[25] M. Mariş. Nonexistence of supersonic traveling waves for nonlinear Schrödinger equations with nonzero conditions at infinity. SIAM J. Math. Anal., 40(3):1076-1103, 2008.

[26] A. Ostrowski. On the Morse-Kuiper theorem. Aequationes Math., 1:66-76, 1968.

[27] L. Pitaevskii. Vortex lines in an imperfect Bose gas. Sov. Phys. JETP, 13(2):451-454, 1961.

[28] Y. Pomeau and S. Rica. Model of superflow with rotons. Phys. Rev. Lett., 71(2):247-250, 1993.

[29] V. S. Shchesnovich and R. A. Kraenkel. Vortices in nonlocal Gross-Pitaevskii equation. J. Phys. A, 37(26):6633-6651, 2004.

[30] S. Yi and L. You. Trapped condensates of atoms with dipole interactions. Phys. Rev. A, 63(5):053607.1-053607.14, 2001.

Laboratoire Jacques-Louis Lions, Université Pierre et Marie Curie, Boîte Courier 187, 75252 Paris Cedex 05, France

E-mail address: delaire@ann.jussieu.fr 